\title{
Cobalt-Mediated Cyclic and Linear 2:1 Cooligomerization of Alkynes with Alkenes: A DFT Study
}

\author{
Vincent Gandon, ${ }^{*}{ }^{\ddagger}$ Nicolas Agenet, ${ }^{\ddagger}$ K. Peter C. Vollhardt, ${ }^{\S}$ Max Malacria, ${ }^{\ddagger}$ and Corinne \\ Aubert ${ }^{*}$, \\ Contribution from the Université Pierre et Marie Curie-Paris 6, Laboratoire de Chimie \\ Organique (UMR CNRS 7611), Institut de Chimie Moléculaire (FR 2769), case. 229, 4 place \\ Jussieu, F-75252 Paris cedex 05, France, and Center for New Directions in Organic \\ Synthesis, Department of Chemistry, University of California at Berkeley, and the Chemical \\ Sciences Division, Lawrence Berkeley National Laboratory, Berkeley, California 94720-1460, \\ USA \\ E-mail: gandon@ccr.jussieu.fr
}

\section{Supporting information}

\section{Complete List of Authors for References with more than 10 Authors}

Ref 22b:

Bianchini, C.; Caulton, K. C.; Chardon, C.; Doublet, M.-L.; Eisenstein, O.; Jackson, S. ; Johnson, T. J. ; Meli, A. ; Peruzzini, M.; Streib, W. E.; Vacca, A.; Vizza, F. Organometallics 1994, 13, 2010-2023.

Ref 29:

Schmidt, M. W.; Baldridge, K. K.; Boatz, J. A.; Elbert, S. T.; Gordon, M. S.; Jensen, J. H.; Koseki, S.; Matsunaga, N.; Nguyen, K. A.; Su, S. J.; Windus, T. L.; Dupuis, M.; Montgomery, J. A. J. Comput. Chem. 1993, $14,1347-1363$.

Ref 32:

Gaussian 03, Revision B.02, Frisch, M. J.; Trucks, G. W.; Schlegel, H. B.; Scuseria, G. E.; Robb, M. A.; Cheeseman, J. R.; Montgomery, Jr., J. A.; Vreven, T.; Kudin, K. N.; Burant, J. C.; Millam, J. M.; Iyengar, S. S.; Tomasi, J.; Barone, V.; Mennucci, B.; Cossi, M.; Scalmani, G.; Rega, N.; Petersson, G. A.; Nakatsuji, H.; Hada, M.; Ehara, M.; Toyota, K.; Fukuda, R.; Hasegawa, J.; Ishida, M.; Nakajima, T.; Honda, Y.; Kitao, O.; Nakai, H.; Klene, M.; Li, X.; Knox, J. E.; Hratchian, H. P.; Cross, J. B.; Bakken, V.; Adamo, C.; Jaramillo, J.; Gomperts, R.; Stratmann, R. E.; Yazyev, O.; Austin, A. J.; Cammi, R.; Pomelli, C.; Ochterski, J. W.; Ayala, P. Y.; Morokuma, K.; Voth, G. A.; Salvador, P.; Dannenberg, J. J.; Zakrzewski, V. G.; Dapprich, S.; Daniels, A. D.; Strain, M. C.;

\footnotetext{
${ }^{\ddagger}$ Laboratoire de Chimie Organique.

${ }^{\S}$ Center for New Directions in Organic Synthesis.
} 
Farkas, O.; Malick, D. K.; Rabuck, A. D.; Raghavachari, K.; Foresman, J. B.; Ortiz, J. V.; Cui, Q.; Baboul, A. G.; Clifford, S.; Cioslowski, J.; Stefanov, B. B.; Liu, G.; Liashenko, A.; Piskorz, P.; Komaromi, I.; Martin, R. L.; Fox, D. J.; Keith, T.; Al-Laham, M. A.; Peng, C. Y.; Nanayakkara, A.; Challacombe, M.; Gill, P. M. W.; Johnson, B.; Chen, W.; Wong, M. W.; Gonzalez, C.; and Pople, J. A.; Gaussian, Inc., Wallingford CT, 2004.

Ref 61b: Álvarez, E.; Gómez, M.; Paneque, M.; Posadas, C. M.; Poveda, M. L.; Rendón, N.; Santos, L. L.; Rojas-Lima, S.; Salazar, V.; Mereiter, K.; Ruiz, C. J. Am. Chem. Soc. 2003, 125, 1478-1479.

\section{Peripheral Discussion on Jahn-Teller Effects in Cobaltacyclopentadienes}

At the B3LYP/6-31G(d,p) level, Dahy and Koga obtained an asymmetric cobaltacyclopentadiene. ${ }^{1}$ Due to a low HOMO-LUMO gap in the $C_{\mathrm{s}}$ geometry, a secondorder Jahn-Teller effect ${ }^{2}$ occurs, resulting in unequal Co-C distances $\left(\mathrm{Co}-\mathrm{C}_{\alpha}=1.831 \AA\right.$; $\mathrm{Co}-\mathrm{C}_{\alpha^{\prime}}=1.905 \AA$ ) and a gain of $2.5 \mathrm{kcal} / \mathrm{mol}$. Although the relatives energies we found for the oxidative coupling are in very good agreement with those of Dahy and Koga, we tried to reproduce this result at the DFT/B3LYP5 level with the 6-31G(d,p) basis set but still, a minimum of $C s$ symmetry was obtained. We thus concluded that this difference was due to the different hybrid functionals used. To make sure that both our basis set and functional could be able to account for a second order Jahn-Teller effect, we optimized some $\alpha, \alpha^{\prime}$ disubstituted cobaltacyclopentadienes. We reasoned that electron-donating substituents could slightly decrease the HOMO-LUMO gap in the $C_{\mathrm{s}}$ geometry and thus promote the distortion. In the parent series, this value is of 0.0816 a.u. When methyl groups are present on the metallacycles, a difference of 0.0787 a.u. between frontier orbitals of the $C_{\mathrm{s}}$-enforced geometry was measured. Without this constraint, the Jahn-Teller effect was effective $\left(\mathrm{Co}-\mathrm{C}_{\alpha}\right.$ $=1.826 \AA ; \mathrm{Co}-\mathrm{C}_{\alpha^{\prime}}=1.927 \AA$ ), resulting in stabilization of the HOMO (from -0.1673 a.u. to -0.1693 a.u.) and increase of the HOMO-LUMO gap to 0.0970 a.u.. On the other hand, electron-withdrawing $\mathrm{B}(\mathrm{OH})_{2}$ groups increased the gap to 0.0885 a.u. and therefore, a quasi Cs structure was obtained $\left(\mathrm{Co}-\mathrm{C}_{\alpha}=1.930 \AA\right.$; $\left.\mathrm{Co}-\mathrm{C}_{\alpha}=1.936 \AA\right)$.

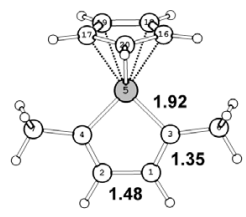

$C_{\mathrm{s}}$-enforced

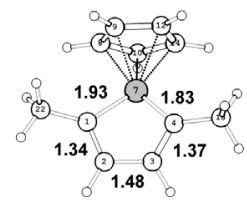

No symmetry constraint

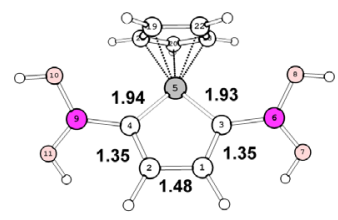

No symmetry constraint

\footnotetext{
${ }^{1}$ Dahy, A. A.; Koga, N. Bull. Chem. Soc. Jpn 2005, 78, 781-791.

${ }^{2}$ Pearson, R. G. Proc. Nat. Acad. Sci. USA 1975, 72, 2104-2106.
} 


\section{$\operatorname{CpCo}\left(\mathrm{C}_{2} \mathrm{H}_{2}\right)$}

Singlet and triplet $\mathrm{CpCo}\left(\mathrm{C}_{2} \mathrm{H}_{2}\right)$ display a linear geometry with respect to the $\mathrm{Cp}$ centroid, cobalt, and the middle of the coordinated $\mathrm{C}-\mathrm{C}$ triple bond. Canonical MO 26 of ${ }^{1}[\mathrm{CpCo}$ (acetylene) $]$ shows some electron density between coordinated acetylene and cobalt, out of the $\mathrm{C}_{\alpha} \mathrm{CoC}_{\alpha}$, plane.

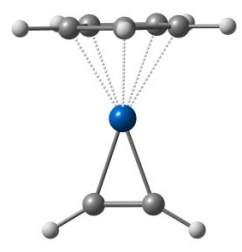

FRONT

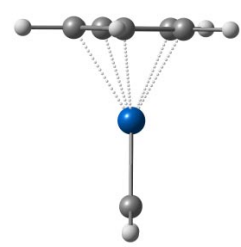

SIDE

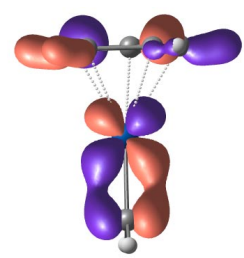

MO 26

$$
\begin{array}{ll}
\text { Triplet: } & \mathrm{CoC}_{\alpha}=1.91 \AA \\
& \mathrm{CC}=1.27 \AA \\
\text { Singlet: } & \mathrm{CoC}_{\alpha}=1.82 \AA \\
& \mathrm{CC}=1.30 \AA
\end{array}
$$

\section{Coordinates of some species involved}

\begin{tabular}{lccccccc}
\hline & \multicolumn{7}{c}{ Atomic Number, $\mathbf{X}, \mathbf{Y}, \mathbf{Z}$} \\
\hline $\mathbf{1}$ & & \multicolumn{7}{c}{$\mathbf{2}$} \\
\hline 6 & -1.619145648 & 0.000000000 & 1.233722399 & 6 & 1.130253682 & 1.202265419 & -1.089772931 \\
6 & -1.592188569 & -1.143010636 & 0.389399816 & 6 & 1.843203422 & 0.332305282 & -0.209171003 \\
6 & -1.592188569 & 1.143010636 & 0.389399816 & 6 & 1.421496061 & 0.586942125 & 1.133319836 \\
6 & -1.614128350 & -0.707608375 & -0.974646184 & 6 & 0.397858551 & 1.562121874 & 1.073083460 \\
6 & -1.614128350 & 0.707608375 & -0.974646184 & 6 & 0.215974594 & 1.939090111 & -0.298392054 \\
1 & -1.611058706 & 0.000000000 & 2.314782869 & 6 & -0.128244216 & -1.868968450 & -1.232666044 \\
1 & -1.549891577 & -2.172679928 & 0.717551166 & 6 & 0.024195053 & -2.203181125 & 0.116910643 \\
1 & -1.549891577 & 2.172679928 & 0.717551166 & 6 & -2.154406093 & -0.037302759 & -0.485210631 \\
1 & -1.604462671 & -1.350069561 & -1.844325984 & 6 & -2.026528559 & -0.343407325 & 0.723735242 \\
1 & -1.604462671 & 1.350069561 & -1.844325984 & 27 & -0.208992482 & -0.177884348 & -0.078930525 \\
6 & 1.461728483 & -1.412800334 & -0.627491695 & 1 & 1.237427802 & 1.252356860 & -2.164291346 \\
6 & 1.461728483 & 1.412800334 & -0.627491695 & 1 & 2.598017043 & -0.384518326 & -0.506348577 \\
6 & 1.464014099 & -1.419538886 & 0.624029646 & 1 & 1.788919369 & 0.093652446 & 2.022325180 \\
6 & 1.464014099 & 1.419538886 & 0.624029646 & 1 & -0.170362791 & 1.943788721 & 1.910175792 \\
27 & 0.190916606 & 0.000000000 & 0.014754923 & 1 & -0.512144555 & 2.651247647 & -0.661974474 \\
1 & 1.750038634 & -1.726336378 & -1.613719355 & 1 & -1.074900201 & -2.034882970 & -1.736144008 \\
\hline
\end{tabular}




\begin{tabular}{|c|c|c|c|c|c|c|c|}
\hline 1 & 1.750038634 & 1.726336378 & -1.613719355 & 1 & 0.735460161 & -1.788684735 & -1.885902721 \\
\hline 1 & 1.754533827 & -1.750487831 & 1.604072495 & 1 & 1.009513533 & -2.387869349 & 0.534438562 \\
\hline \multirow[t]{3}{*}{1} & 1.754533827 & 1.750487831 & 1.604072495 & 1 & -0.801996135 & -2.632437842 & 0.673889408 \\
\hline & & & & 1 & -2.693116648 & 0.196402292 & -1.385489405 \\
\hline & & & & 1 & -2.364641592 & -0.587751549 & 1.714287596 \\
\hline 3 & & & & ${ }^{3}(3)$ & & & \\
\hline 6 & 2.306568862 & -0.524213100 & 0.777130948 & 6 & 2.714045654 & 0.740057480 & -0.149601304 \\
\hline 6 & 2.311907762 & -0.568661131 & -0.708694275 & 6 & 2.713974091 & -0.739955685 & -0.149641162 \\
\hline 6 & 1.090625837 & -0.382212499 & 1.328909045 & 6 & 1.521579063 & 1.344778131 & -0.021111828 \\
\hline 6 & 1.099020105 & -0.465264889 & -1.274731219 & 6 & 1.521524536 & -1.344620086 & -0.020769521 \\
\hline 6 & -1.437640539 & 1.000425538 & -0.047661444 & 27 & 0.113952027 & 0.000052905 & 0.125797759 \\
\hline 6 & -1.855023492 & 0.314568830 & 1.149718867 & 6 & -1.743889786 & 1.146170633 & 0.714385213 \\
\hline 6 & -1.861558261 & 0.204110496 & -1.172252859 & 6 & -1.744045922 & -1.146254599 & 0.714435692 \\
\hline 6 & -2.399203809 & -0.921063363 & 0.768343141 & 6 & -1.872480785 & 0.707276658 & -0.643641329 \\
\hline 6 & -2.403568251 & -0.988865570 & -0.671850053 & 6 & -1.872570768 & -0.707363657 & -0.643685655 \\
\hline 27 & -0.287832778 & -0.651360763 & 0.031396524 & 6 & -1.700628071 & -0.000010591 & 1.549545931 \\
\hline 1 & 3.236787427 & -0.587874281 & 1.345926416 & 1 & 3.650947484 & 1.292761234 & -0.250904633 \\
\hline 1 & 3.246487690 & -0.660205203 & -1.266303160 & 1 & 3.650790318 & -1.292807350 & -0.251654845 \\
\hline 1 & 0.924988092 & -0.292518619 & 2.402972658 & 1 & 1.384681112 & 2.424317692 & -0.007215432 \\
\hline 1 & 0.939435795 & -0.441678718 & -2.353159063 & 1 & 1.384537346 & -2.424146914 & -0.007078700 \\
\hline 1 & -0.991842040 & 1.983529287 & -0.095527277 & 1 & -1.701421140 & 2.175479079 & 1.044891216 \\
\hline 1 & -1.682470400 & 0.655749259 & 2.160759217 & 1 & -1.701527363 & -2.175551514 & 1.044905015 \\
\hline 1 & -1.695053438 & 0.446971505 & -2.212294413 & 1 & -1.935919539 & 1.347768840 & -1.513073577 \\
\hline 1 & -2.736699825 & -1.702183823 & 1.437154688 & 1 & -1.936043632 & -1.347896110 & -1.512983477 \\
\hline 1 & -2.743428734 & -1.830252954 & -1.261837742 & 1 & -1.586504624 & -0.000056144 & 2.625400640 \\
\hline 4 & & & & ${ }^{3}(4)$ & & & \\
\hline 6 & 1.979185709 & 0.737933929 & -0.481544465 & 6 & 2.107968899 & 0.704497499 & -0.447922704 \\
\hline 6 & 2.102359253 & -0.707460697 & -0.116746321 & 6 & 2.190817764 & -0.790216520 & -0.354826529 \\
\hline 6 & 0.726840861 & 1.201174022 & -0.666100227 & 6 & 0.888318797 & 1.268383732 & -0.484528855 \\
\hline 6 & 0.755120694 & -1.359491074 & -0.436543108 & 6 & 0.815287483 & -1.364638860 & -0.731757218 \\
\hline 27 & -0.649050324 & -0.064174249 & -0.670091714 & 27 & -0.547101365 & 0.007444924 & -0.354518172 \\
\hline 6 & -2.150701658 & 1.145795372 & 0.239387067 & 6 & -2.433685948 & 1.189995943 & 0.218104699 \\
\hline 6 & -2.277290387 & -1.175606161 & 0.237833610 & 6 & -2.337068917 & -1.083679026 & 0.504591857 \\
\hline 6 & -2.705737335 & 0.739525973 & -0.985645825 & 6 & -2.632588734 & 0.583140197 & -1.058688013 \\
\hline 6 & -2.775491883 & -0.699385824 & -0.985568210 & 6 & -2.563716281 & -0.821560993 & -0.886378587 \\
\hline 6 & -1.809678779 & -0.040045090 & 0.985607928 & 6 & -2.283344256 & 0.158888160 & 1.184209373 \\
\hline 1 & 2.872274792 & 1.361048496 & -0.584244073 & 1 & 3.027593049 & 1.296891063 & -0.462504031 \\
\hline 1 & 2.331160707 & -0.801907222 & 0.956191941 & 1 & 2.461618555 & -1.082162895 & 0.670695991 \\
\hline 1 & 2.944068696 & -1.194783282 & -0.635847100 & 1 & 2.988463471 & -1.199906911 & -0.995363630 \\
\hline 1 & 0.540950179 & 2.260987955 & -0.862652256 & 1 & 0.751849085 & 2.348949436 & -0.542326240 \\
\hline 1 & 0.740442420 & -1.701098282 & -1.491230597 & 1 & 0.714750621 & -1.472144914 & -1.818830700 \\
\hline 1 & 0.500565176 & -2.210313641 & 0.202186933 & 1 & 0.570208432 & -2.318750471 & -0.257075532 \\
\hline 1 & -1.928688350 & 2.160254713 & 0.539456940 & 1 & -2.409624498 & 2.253414644 & 0.416583349 \\
\hline 1 & -2.173731620 & -2.211534470 & 0.528205946 & 1 & -2.239537553 & -2.061991079 & 0.956252272 \\
\hline 1 & -3.004304337 & 1.387006690 & -1.799134978 & 1 & -2.782896964 & 1.106418526 & -1.993552809 \\
\hline 1 & -3.128214434 & -1.309766855 & -1.807308837 & 1 & -2.670632732 & -1.567910665 & -1.662330779 \\
\hline 1 & -1.374838380 & -0.062930300 & 1.974976346 & 1 & -2.101437909 & 0.300168212 & 2.241355259 \\
\hline 5 & & & & 6 & & & \\
\hline 6 & 1.338015420 & -0.035723907 & -1.305816831 & 6 & 0.146752945 & -0.476381309 & 1.294615322 \\
\hline 6 & 2.316105100 & 0.997754857 & -0.773216151 & 6 & -1.756015970 & -2.808503197 & -0.570949541 \\
\hline
\end{tabular}




\begin{tabular}{|c|c|c|c|c|c|c|c|}
\hline 6 & 1.231730048 & -1.328111758 & -0.713644086 & 6 & 0.206494821 & -0.158932999 & -0.131961900 \\
\hline 27 & -0.226901019 & -0.177135287 & -0.000007456 & 6 & -2.362745600 & -3.106577224 & 0.633841025 \\
\hline 6 & 2.316093300 & 0.997759503 & 0.773213675 & 6 & -1.054905787 & -0.903090568 & 1.722425138 \\
\hline 6 & 1.231730811 & -1.328109270 & 0.713638283 & 6 & -0.939730579 & -0.383973891 & -0.797054427 \\
\hline 6 & 1.338021908 & -0.035725608 & 1.305815413 & 6 & -3.251200053 & 0.901172998 & 0.152686111 \\
\hline 1 & 1.110944229 & 0.034109911 & -2.368464193 & 6 & -3.556088346 & 0.401165113 & 1.454251087 \\
\hline 1 & 2.036225858 & 1.985103141 & -1.156711589 & 6 & -3.949467035 & 0.087832160 & -0.805098943 \\
\hline 1 & 3.325064789 & 0.795334979 & -1.164662752 & 6 & -4.369723432 & -0.751657511 & 1.300902613 \\
\hline 1 & 0.985701037 & -2.222652212 & -1.278110304 & 6 & -4.622583460 & -0.925032585 & -0.099149948 \\
\hline 1 & 2.036168906 & 1.985094821 & 1.156696007 & 27 & -2.400951552 & -1.004147163 & 0.342048837 \\
\hline 1 & 3.325057064 & 0.795378064 & 1.164671705 & 1 & -2.307118113 & -2.855734428 & -1.505675826 \\
\hline 1 & 0.985671037 & -2.222640737 & 1.278115649 & 1 & -0.677467396 & -2.791924395 & -0.648080384 \\
\hline 1 & 1.110937285 & 0.034101114 & 2.368461854 & 1 & 1.017960970 & -0.372810600 & 1.944011823 \\
\hline 6 & -1.819615366 & 0.576934297 & 1.148014842 & 1 & -1.764499270 & -3.325233472 & 1.510112740 \\
\hline 6 & -2.216022042 & -0.724418246 & 0.709106249 & 1 & -3.407697874 & -3.396173070 & 0.679012506 \\
\hline 6 & -1.604061124 & 1.399388006 & 0.000003563 & 1 & 1.114250378 & 0.223162168 & -0.601537705 \\
\hline 6 & -2.216024460 & -0.724417449 & -0.709104389 & 1 & -1.269015734 & -1.176557797 & 2.756663779 \\
\hline 6 & -1.819622949 & 0.576938402 & -1.148009055 & 1 & -1.070643839 & -0.216494686 & -1.866122114 \\
\hline 1 & -1.708987719 & 0.888258399 & 2.178006779 & 1 & -2.643959431 & 1.766656776 & -0.067166565 \\
\hline 1 & -2.441311983 & -1.566827142 & 1.349285865 & 1 & -3.186252687 & 0.804828964 & 2.385395760 \\
\hline 1 & -1.321737502 & 2.442605153 & 0.000003034 & 1 & -3.915872913 & 0.206644802 & -1.879420990 \\
\hline 1 & -2.441329233 & -1.566821449 & -1.349287648 & 1 & -4.750107344 & -1.374214511 & 2.099097826 \\
\hline 1 & -1.709010324 & 0.888271881 & -2.177998464 & 1 & -5.194207699 & -1.731574573 & -0.540931222 \\
\hline 7 & & & & ${ }^{3}(7)$ & & & \\
\hline 6 & -2.005556392 & 0.669715119 & -0.676520911 & 6 & -2.154867003 & 0.441249461 & -0.578320488 \\
\hline 6 & -1.637378735 & -0.676586298 & -0.164018252 & 6 & -2.155790525 & -1.027832913 & -0.542591746 \\
\hline 6 & -0.764522041 & -1.502037483 & -0.830308371 & 6 & -1.333954012 & -1.799990373 & -1.278321674 \\
\hline 6 & -0.230649836 & -1.224063643 & -2.230357115 & 6 & -0.350627694 & -1.257593467 & -2.291999222 \\
\hline 6 & 0.919772423 & -0.246961462 & -1.996502286 & 6 & 0.941544065 & -0.698022867 & -1.698021344 \\
\hline 27 & 0.461246068 & 0.235013936 & -0.146608554 & 27 & 0.695615322 & 0.508504423 & -0.159588980 \\
\hline 6 & -0.885063020 & 1.381030952 & -0.844207268 & 6 & -1.048819505 & 1.209200196 & -0.564997997 \\
\hline 1 & -3.043474475 & 0.976912942 & -0.816702577 & 1 & -3.142001468 & 0.916792990 & -0.605864262 \\
\hline 1 & -2.041088554 & -1.016863947 & 0.791621337 & 1 & -2.906871662 & -1.506686208 & 0.087550984 \\
\hline 1 & -0.482294435 & -2.439279534 & -0.354158493 & 1 & -1.404974538 & -2.882630698 & -1.177051930 \\
\hline 1 & -1.020067406 & -0.768846717 & -2.836495455 & 1 & -0.843539293 & -0.453602520 & -2.853102765 \\
\hline 1 & 0.074751929 & -2.159961624 & -2.713591380 & 1 & -0.104111747 & -2.051900669 & -3.013181338 \\
\hline 1 & 0.933788440 & 0.609279535 & -2.674499753 & 1 & 1.491617803 & -0.094760979 & -2.431868388 \\
\hline 1 & 1.896379505 & -0.738532107 & -1.988802848 & 1 & 1.603694428 & -1.485893540 & -1.319093744 \\
\hline 1 & -0.819353958 & 2.406664873 & -1.205712691 & 1 & -1.157240663 & 2.294096957 & -0.640797710 \\
\hline 6 & 1.738259553 & -0.547430962 & 1.535633934 & 6 & 2.027856912 & -0.233796601 & 1.498113700 \\
\hline 6 & 2.526482886 & 0.138159170 & 0.591942363 & 6 & 2.838617937 & 0.526343800 & 0.594045552 \\
\hline 6 & 2.026172863 & 1.476811305 & 0.486333183 & 6 & 2.412116026 & 1.878874713 & 0.646289449 \\
\hline 6 & 0.986670384 & 1.628022411 & 1.465337164 & 6 & 1.313918269 & 1.951023009 & 1.541247523 \\
\hline 6 & 0.787039946 & 0.380608163 & 2.086623310 & 6 & 1.087844379 & 0.647351187 & 2.083165889 \\
\hline 1 & 1.834018996 & -1.590487689 & 1.807769067 & 1 & 2.116405779 & -1.295644283 & 1.690794625 \\
\hline 1 & 3.318166553 & -0.288735802 & -0.008096130 & 1 & 3.645927336 & 0.135809962 & -0.013112562 \\
\hline 1 & 2.424211282 & 2.258673567 & -0.145722565 & 1 & 2.818253407 & 2.698390675 & 0.066319157 \\
\hline 1 & 0.409504556 & 2.525825516 & 1.639152417 & 1 & 0.741413397 & 2.840033681 & 1.774932797 \\
\hline 1 & 0.042775467 & 0.154344777 & 2.838536872 & 1 & 0.317765052 & 0.381959064 & 2.796099476 \\
\hline
\end{tabular}




\begin{tabular}{|c|c|c|c|c|c|c|c|}
\hline 6 & 1.430751752 & 3.067336085 & -3.868697652 & 6 & -1.968858649 & -0.623819832 & 1.291449509 \\
\hline 6 & 1.171613470 & 1.784476645 & -3.128056531 & 6 & -1.893124311 & 0.725175892 & 1.305062962 \\
\hline 6 & 0.407733674 & 3.552308497 & -4.575925300 & 6 & -1.250477491 & -1.242878560 & 0.161644010 \\
\hline 6 & -0.044221703 & 1.147599650 & -3.809635474 & 6 & -1.192524490 & 1.267143947 & 0.108157842 \\
\hline 27 & -1.276457835 & 2.601409033 & -4.436432385 & 6 & 2.487215198 & -0.052672701 & -0.850670258 \\
\hline 6 & -2.050828070 & 4.282877125 & -3.292226029 & 6 & 2.207703739 & -1.200843056 & -0.059898558 \\
\hline 6 & -2.847307020 & 2.137401010 & -2.994162041 & 6 & 2.125283937 & 1.099015934 & -0.113555187 \\
\hline 6 & -3.028634913 & 4.037716807 & -4.287835393 & 6 & 1.725266413 & -0.755746157 & 1.210213466 \\
\hline 6 & -3.517663811 & 2.712525365 & -4.099569102 & 6 & 1.658148798 & 0.665221741 & 1.178665309 \\
\hline 6 & -1.917108036 & 3.103331923 & -2.496555100 & 27 & 0.280594160 & -0.070528464 & -0.186109570 \\
\hline 1 & 2.400721452 & 3.567778062 & -3.803344186 & 6 & -1.468881022 & -0.655990953 & -1.232370164 \\
\hline 1 & 0.977291638 & 1.988189649 & -2.061850412 & 6 & -1.977019432 & 0.788738558 & -1.144410807 \\
\hline 1 & 2.041677017 & 1.107438750 & -3.148487880 & 1 & -2.486109452 & -1.221349497 & 2.039618719 \\
\hline 1 & 0.474519353 & 4.485667866 & -5.141630210 & 1 & -2.362008692 & 1.352640796 & 2.062725158 \\
\hline 1 & 0.279446720 & 0.623859894 & -4.712875053 & 1 & -1.083518827 & -2.320093240 & 0.191977849 \\
\hline 1 & -0.597078336 & 0.449712616 & -3.170840089 & 1 & -0.940239219 & 2.329076949 & 0.126091520 \\
\hline 1 & -1.472084007 & 5.188823757 & -3.174471723 & 1 & 2.835739794 & -0.061511813 & -1.876805804 \\
\hline 1 & -2.990876400 & 1.135777307 & -2.610961247 & 1 & 2.357216957 & -2.231518770 & -0.356624904 \\
\hline 1 & -3.327918704 & 4.721712295 & -5.071389156 & 1 & 2.204007648 & 2.125570608 & -0.447218737 \\
\hline 1 & -4.243484733 & 2.212771185 & -4.728893166 & 1 & 1.434852083 & -1.386681055 & 2.039979562 \\
\hline 1 & -1.258274526 & 2.980478055 & -1.648170033 & 1 & 1.334336579 & 1.306540767 & 1.987476028 \\
\hline 6 & -1.068838403 & 2.561387784 & -6.475094635 & 1 & -0.424630555 & -0.553482912 & -1.742229651 \\
\hline 6 & -1.512713502 & 1.460444212 & -6.118505279 & 1 & -1.950028851 & -1.332725828 & -1.948610968 \\
\hline 1 & -0.692877152 & 3.348025027 & -7.099241643 & 1 & -1.695488875 & 1.348608587 & -2.042952344 \\
\hline 1 & -1.852244926 & 0.443538401 & -6.134187281 & 1 & -3.069373440 & 0.847188060 & -1.028321984 \\
\hline 10 & & & & 11 & & & \\
\hline 6 & -2.759962272 & -0.676843393 & -0.121960713 & 6 & -2.002930629 & -0.564129260 & 0.938708553 \\
\hline 6 & -2.756509067 & 0.690783338 & -0.121898651 & 6 & -2.495707874 & -1.261244351 & -0.244972777 \\
\hline 6 & -1.409929994 & -1.202922679 & 0.013841579 & 6 & -1.763260451 & -1.617562717 & -1.316777602 \\
\hline 6 & -1.403837391 & 1.210026072 & 0.013951078 & 6 & -0.286439096 & -1.376660032 & -1.407763826 \\
\hline 27 & -0.122979253 & 0.000302744 & 0.171301507 & 6 & 0.582702845 & -1.971722849 & -0.340180847 \\
\hline 6 & 1.695507979 & -1.152435228 & 0.714045670 & 27 & 0.764331578 & -0.093533896 & 0.012307873 \\
\hline 6 & 1.701305869 & 1.143793592 & 0.714149872 & 6 & -0.754172880 & -0.088320824 & 1.154124164 \\
\hline 6 & 1.860424930 & -0.713903315 & -0.634194118 & 1 & -2.756389820 & -0.396358630 & 1.711929096 \\
\hline 6 & 1.864006487 & 0.704556824 & -0.634129749 & 1 & -3.558449312 & -1.497993205 & -0.256348054 \\
\hline 6 & 1.628876681 & -0.004183306 & 1.552574887 & 1 & -2.236029101 & -2.096094097 & -2.170425939 \\
\hline 1 & -3.657487087 & -1.288194080 & -0.213143252 & 1 & -0.228951817 & -0.182376732 & -1.334398341 \\
\hline 1 & -3.650935163 & 1.306666905 & -0.213025499 & 1 & 0.112338592 & -1.522315533 & -2.415628157 \\
\hline 1 & -1.282961227 & -2.291772828 & 0.001623659 & 1 & 1.509968265 & -2.424834682 & -0.689375145 \\
\hline 1 & -1.271371665 & 2.298222263 & 0.001831950 & 1 & 0.073536122 & -2.558435161 & 0.419136481 \\
\hline 1 & 1.653837995 & -2.182858311 & 1.044621135 & 1 & -0.598367677 & 0.400138619 & 2.118890659 \\
\hline 1 & 1.664839864 & 2.174383960 & 1.044818865 & 6 & 1.579521535 & 1.762169437 & 0.792248168 \\
\hline 1 & 1.948654299 & -1.351619751 & -1.504553381 & 6 & 2.148911570 & 0.589348503 & 1.400887053 \\
\hline 1 & 1.955455330 & 1.341898570 & -1.504431151 & 6 & 2.886608304 & -0.107167001 & 0.395504350 \\
\hline \multirow[t]{6}{*}{1} & 1.512471627 & -0.003938215 & 2.628559221 & 6 & 2.709339986 & 0.576588565 & -0.831118141 \\
\hline & & & & 6 & 1.909922705 & 1.739460234 & -0.577531474 \\
\hline & & & & 1 & 0.957446940 & 2.494906237 & 1.286641697 \\
\hline & & & & 1 & 2.086609359 & 0.318789139 & 2.445590775 \\
\hline & & & & 1 & 3.428081877 & -1.031875192 & 0.539229466 \\
\hline & & & & 1 & 3.106678647 & 0.277000269 & -1.791894859 \\
\hline
\end{tabular}




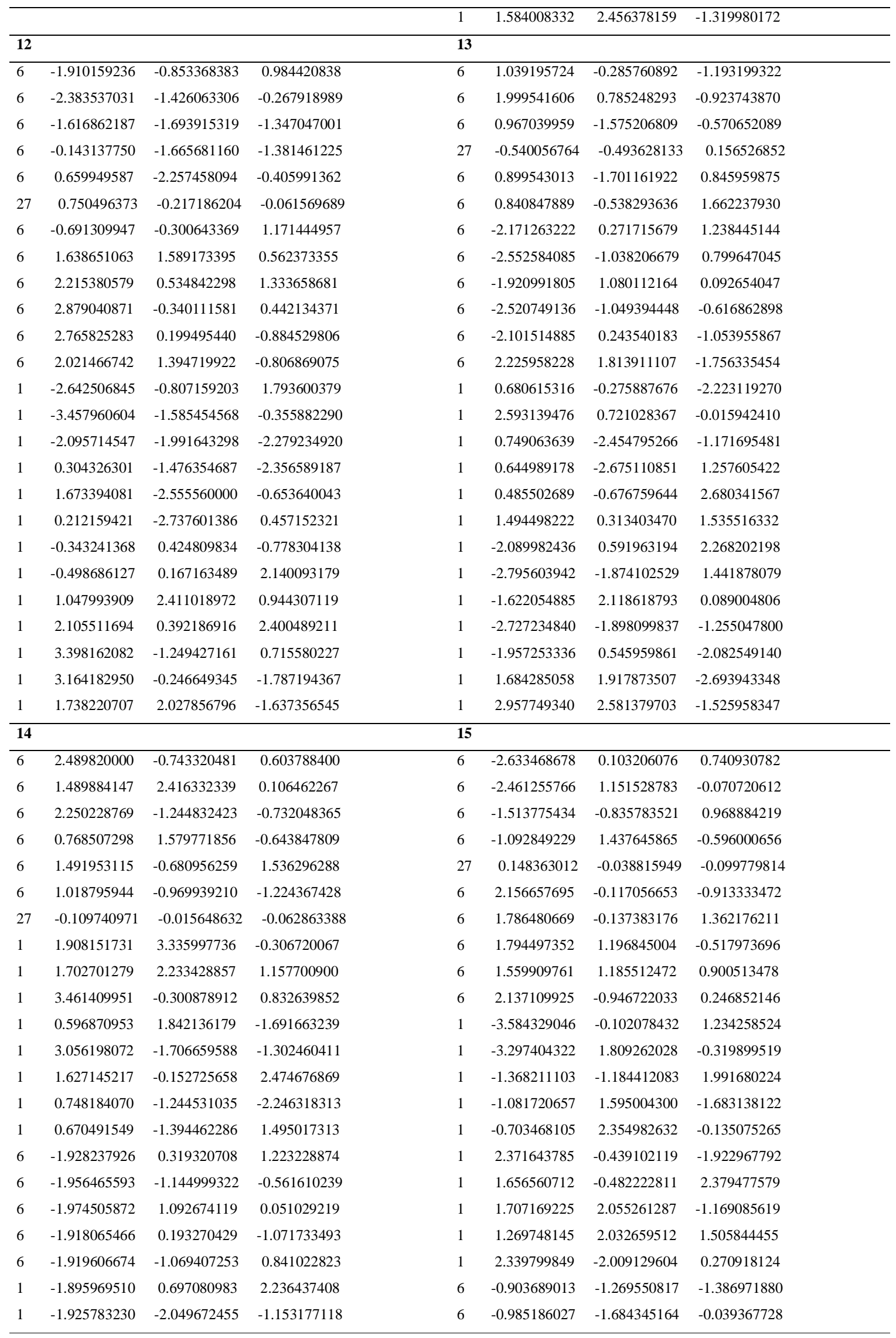




\begin{tabular}{|c|c|c|c|c|c|c|c|}
\hline 1 & -1.965562477 & 2.171369601 & -0.003527881 & 1 & -0.333661159 & -1.883471834 & -2.078380519 \\
\hline 1 & -1.947944942 & 0.479656426 & -2.114220886 & 1 & -1.689122838 & -0.668150162 & -1.829422402 \\
\hline 1 & -1.876698434 & -1.910406720 & 1.521595426 & 1 & -0.393601753 & -2.539763600 & 0.281002353 \\
\hline 16 & & & & 17 & & & \\
\hline 6 & 2.372433000 & -1.928795000 & 0.780624000 & 6 & 1.416369000 & 2.381900000 & -0.979696000 \\
\hline 6 & -1.180440000 & -1.367150000 & 0.073717000 & 6 & 1.897647000 & 2.457142000 & 0.385726000 \\
\hline 6 & 2.338110000 & -1.989812000 & -0.688520000 & 6 & 0.745913000 & 1.255606000 & -1.324957000 \\
\hline 6 & -1.744555000 & -0.880808000 & 1.265036000 & 6 & 1.271215000 & 1.777524000 & 1.383715000 \\
\hline 6 & 1.632709000 & -0.940223000 & 1.315815000 & 6 & 2.589920000 & -1.157805000 & 0.495657000 \\
\hline 6 & 1.566856000 & -1.050634000 & -1.266082000 & 6 & 2.305058000 & -1.249458000 & -0.875003000 \\
\hline 6 & 1.831918000 & 1.745179000 & -0.574119000 & 6 & 1.504058000 & -1.765959000 & 1.226607000 \\
\hline 6 & 1.541272000 & 1.888660000 & 0.822534000 & 6 & 1.001736000 & -1.845787000 & -1.012408000 \\
\hline 6 & 0.599545000 & 1.901705000 & -1.295794000 & 6 & 0.561475000 & -2.236132000 & 0.299176000 \\
\hline 6 & 0.143218000 & 2.059218000 & 0.965816000 & 27 & 0.672281000 & -0.076565000 & 0.000948000 \\
\hline 6 & -0.427211000 & 2.068012000 & -0.347297000 & 1 & 1.623414000 & 3.199201000 & -1.671500000 \\
\hline 27 & 0.690400000 & 0.125385000 & 0.004351000 & 1 & 2.812857000 & 3.011471000 & 0.601407000 \\
\hline 1 & 2.950210000 & -2.645989000 & 1.367517000 & 1 & 0.333458000 & 1.142196000 & -2.328658000 \\
\hline 1 & -0.300443000 & -2.002975000 & 0.108582000 & 1 & 1.685953000 & 1.688629000 & 2.381911000 \\
\hline 1 & 2.895600000 & -2.749764000 & -1.239928000 & 1 & 3.462540000 & -0.693814000 & 0.934550000 \\
\hline 1 & -1.238627000 & -1.069888000 & 2.205991000 & 1 & 2.910450000 & -0.860018000 & -1.681362000 \\
\hline 1 & 1.565715000 & -0.761850000 & 2.391364000 & 1 & 1.433693000 & -1.844164000 & 2.303283000 \\
\hline 1 & 1.451292000 & -0.960106000 & -2.348218000 & 1 & 0.499279000 & -2.072620000 & -1.942301000 \\
\hline 1 & 2.811846000 & 1.597118000 & -1.003768000 & 1 & -0.383044000 & -2.707180000 & 0.529808000 \\
\hline 1 & 2.265652000 & 1.819380000 & 1.621158000 & 6 & -1.256922000 & 0.034548000 & -0.004181000 \\
\hline 1 & 0.479503000 & 1.840495000 & -2.368290000 & 6 & -2.029923000 & -0.125990000 & -1.165265000 \\
\hline 1 & -0.398307000 & 2.163330000 & 1.895383000 & 6 & -3.426618000 & -0.111385000 & -1.120327000 \\
\hline 1 & -1.485085000 & 2.123882000 & -0.566580000 & 6 & -4.091067000 & 0.068363000 & 0.094706000 \\
\hline 6 & -2.959589000 & -0.190256000 & 1.234822000 & 6 & -3.343293000 & 0.224060000 & 1.261493000 \\
\hline 6 & -3.611973000 & 0.011510000 & 0.016831000 & 6 & -1.944503000 & 0.202387000 & 1.210448000 \\
\hline 6 & -3.050757000 & -0.457564000 & -1.177564000 & 1 & -1.542005000 & -0.267529000 & -2.126579000 \\
\hline 6 & -1.837439000 & -1.142230000 & -1.150207000 & 1 & -3.996500000 & -0.238189000 & -2.038034000 \\
\hline 1 & -3.400813000 & 0.178243000 & 2.156255000 & 1 & -5.176875000 & 0.083894000 & 0.131111000 \\
\hline 1 & -4.563203000 & 0.536618000 & -0.005148000 & 1 & -3.844564000 & 0.357868000 & 2.217382000 \\
\hline 1 & -3.564595000 & -0.293116000 & -2.120213000 & 1 & -1.394875000 & 0.307022000 & 2.144700000 \\
\hline 1 & -1.394111000 & -1.521653000 & -2.065249000 & 1 & 0.218225000 & 1.506389000 & 1.264541000 \\
\hline 18 & & & & 19 & & & \\
\hline 6 & 0.801393000 & 2.615055000 & 0.786079000 & 6 & -0.469388000 & 0.238147000 & -1.187301000 \\
\hline 6 & 1.523434000 & 2.596305000 & -0.338891000 & 6 & 0.270645000 & 1.467003000 & -1.220370000 \\
\hline 6 & 0.140730000 & 1.356904000 & 1.217235000 & 27 & 1.225632000 & 0.212024000 & -0.003114000 \\
\hline 6 & 1.731365000 & 1.275614000 & -1.010696000 & 6 & 0.617349000 & 2.119589000 & -0.001456000 \\
\hline 27 & 0.657479000 & -0.119333000 & -0.073710000 & 6 & 0.262330000 & 1.518157000 & 1.236423000 \\
\hline 6 & 0.147075000 & -2.241828000 & -0.359754000 & 6 & 2.489775000 & -0.698725000 & 1.404895000 \\
\hline 6 & 1.747445000 & -1.532213000 & 1.133105000 & 6 & 3.338408000 & -0.035658000 & 0.460486000 \\
\hline 6 & 1.298228000 & -1.886944000 & -1.113550000 & 6 & 1.729978000 & -1.687508000 & 0.716339000 \\
\hline 6 & 2.288665000 & -1.438914000 & -0.182330000 & 6 & 3.096508000 & -0.602085000 & -0.816207000 \\
\hline 6 & 0.419928000 & -2.032036000 & 1.019282000 & 6 & 2.084554000 & -1.596766000 & -0.667597000 \\
\hline 1 & 0.664848000 & 3.518757000 & 1.382011000 & 1 & -0.310505000 & -0.386645000 & -2.066877000 \\
\hline 1 & 1.974669000 & 3.506592000 & -0.740488000 & 1 & 0.756874000 & 1.794936000 & -2.136071000 \\
\hline 1 & 0.176700000 & 1.142546000 & 2.287149000 & 1 & 1.347389000 & 2.924672000 & -0.040930000 \\
\hline 1 & 1.457394000 & 1.290014000 & -2.076093000 & 1 & 0.786217000 & 1.870767000 & 2.121278000 \\
\hline
\end{tabular}




\begin{tabular}{|c|c|c|c|c|c|c|c|}
\hline 1 & 2.792816000 & 1.004001000 & -0.968255000 & 1 & -0.723125000 & 1.110585000 & 1.422783000 \\
\hline 1 & -0.805484000 & -2.549799000 & -0.770197000 & 1 & 2.435751000 & -0.483348000 & 2.463114000 \\
\hline 1 & 2.253093000 & -1.253448000 & 2.047107000 & 1 & 4.024825000 & 0.769689000 & 0.684492000 \\
\hline 1 & 1.406602000 & -1.940214000 & -2.187386000 & 1 & 1.005224000 & -2.359884000 & 1.152351000 \\
\hline 1 & 3.284450000 & -1.101197000 & -0.430063000 & 1 & 3.556799000 & -0.300618000 & -1.747396000 \\
\hline 1 & -0.279411000 & -2.176878000 & 1.830344000 & 1 & 1.665473000 & -2.193530000 & -1.466515000 \\
\hline 6 & -2.077523000 & 0.144389000 & 1.290853000 & 6 & -1.788991000 & -0.013671000 & -0.562008000 \\
\hline 6 & -3.133253000 & -0.445128000 & 0.638244000 & 6 & -2.247786000 & -1.338873000 & -0.433301000 \\
\hline 6 & -3.241430000 & -0.373348000 & -0.774813000 & 6 & -3.499066000 & -1.621614000 & 0.106480000 \\
\hline 6 & -2.298317000 & 0.328048000 & -1.493521000 & 6 & -4.334199000 & -0.582997000 & 0.530328000 \\
\hline 6 & -1.213072000 & 0.973886000 & -0.842544000 & 6 & -3.902902000 & 0.736849000 & 0.395849000 \\
\hline 6 & -1.079963000 & 0.889448000 & 0.577155000 & 6 & -2.649083000 & 1.019814000 & -0.149245000 \\
\hline 1 & -1.997044000 & 0.084740000 & 2.373235000 & 1 & -1.603198000 & -2.151680000 & -0.759267000 \\
\hline 1 & -3.895971000 & -0.968931000 & 1.208410000 & 1 & -3.826289000 & -2.654065000 & 0.196365000 \\
\hline 1 & -4.079472000 & -0.846454000 & -1.278126000 & 1 & -5.311675000 & -0.801606000 & 0.950384000 \\
\hline 1 & -2.396354000 & 0.430671000 & -2.570898000 & 1 & -4.548659000 & 1.554413000 & 0.705197000 \\
\hline 1 & -0.636997000 & 1.706172000 & -1.391691000 & 1 & -2.335953000 & 2.051694000 & -0.274719000 \\
\hline 20 & & & & 21 & & & \\
\hline 6 & 3.479863838 & -1.030636591 & 1.572839054 & 6 & 1.361480353 & 0.030535894 & -1.153326066 \\
\hline 6 & 3.054814067 & -1.447226602 & 0.159041246 & 6 & 2.423195866 & 0.956750588 & -0.580206884 \\
\hline 6 & 3.697100622 & -2.779374230 & -0.275314253 & 6 & 1.129639545 & -1.209480480 & -0.524376282 \\
\hline 6 & 5.158886156 & -2.855149172 & 0.102986526 & 27 & -0.389170216 & 0.068324733 & -0.070619054 \\
\hline 6 & 5.618983182 & -2.248592094 & 1.213653244 & 6 & 2.499241702 & 0.894490450 & 0.958390958 \\
\hline 6 & 4.723214036 & -1.452058101 & 2.072392720 & 6 & 1.751285323 & -1.479395422 & 0.784059369 \\
\hline 27 & 4.888001260 & 0.436438712 & 1.318780937 & 6 & 2.397804440 & -0.521388152 & 1.477740946 \\
\hline 6 & 4.433330778 & 2.447823982 & 2.481808428 & 1 & 1.161022951 & 0.123102763 & -2.219108606 \\
\hline 6 & 4.457573397 & 2.626272270 & 1.070474317 & 1 & 2.255656563 & 1.990510197 & -0.903150014 \\
\hline 6 & 5.769493375 & 2.294562151 & 0.603677528 & 1 & 3.397616958 & 0.653085369 & -0.999074026 \\
\hline 6 & 6.544205595 & 1.904403475 & 1.740866392 & 1 & 0.730098357 & -2.050999872 & -1.085063707 \\
\hline 6 & 5.717905119 & 2.004348210 & 2.894463321 & 1 & 1.678427438 & 1.495349652 & 1.391912567 \\
\hline 1 & 2.702595227 & -0.708863705 & 2.261455105 & 1 & 3.424565097 & 1.369012343 & 1.308273933 \\
\hline 1 & 1.961924717 & -1.520694455 & 0.114099340 & 1 & 1.653359340 & -2.482231826 & 1.194194441 \\
\hline 1 & 3.325120024 & -0.673209227 & -0.578850197 & 1 & 2.819427639 & -0.743513170 & 2.455828202 \\
\hline 1 & 3.166927651 & -3.620837939 & 0.202086746 & 6 & -2.175032503 & 0.847633269 & 0.911947289 \\
\hline 1 & 3.569817272 & -2.916692668 & -1.355940893 & 6 & -2.445198035 & -0.531455453 & 0.651363524 \\
\hline 1 & 5.822873820 & -3.465799406 & -0.505462458 & 6 & -2.079861978 & 1.514424562 & -0.350964074 \\
\hline 1 & 6.659344286 & -2.353404191 & 1.514542596 & 6 & -2.523257140 & -0.707995365 & -0.757932902 \\
\hline 1 & 4.931864244 & -1.417707177 & 3.139446796 & 6 & -2.298234090 & 0.551247322 & -1.375175010 \\
\hline 1 & 3.577487211 & 2.608996191 & 3.126118170 & 1 & -2.121404196 & 1.314844446 & 1.887941308 \\
\hline 1 & 3.637805378 & 2.986091905 & 0.459976213 & 1 & -2.590457835 & -1.301849262 & 1.399372920 \\
\hline 1 & 6.131383654 & 2.392637751 & -0.412680628 & 1 & -1.906655534 & 2.573653546 & -0.499651007 \\
\hline 1 & 7.587881630 & 1.613108462 & 1.727973043 & 1 & -2.712159043 & -1.643680282 & -1.270328251 \\
\hline 1 & 6.014954110 & 1.768156270 & 3.909270301 & 1 & -2.280061738 & 0.745011309 & -2.440883107 \\
\hline \multicolumn{4}{|c|}{$\mathrm{TS}_{1-3}$} & \multicolumn{4}{|c|}{$\mathrm{TS}_{6-14}$} \\
\hline 6 & -1.928782525 & 1.002339556 & -0.462319712 & 6 & -2.550188865 & -0.797872539 & -0.601669060 \\
\hline 6 & -1.928782525 & -1.002339556 & -0.462319712 & 6 & -2.565620087 & -0.475910578 & 0.816653710 \\
\hline 6 & -1.352300710 & 1.480394574 & 0.574673881 & 6 & -1.354507306 & -0.621655258 & -1.204847023 \\
\hline 6 & -1.352300710 & -1.480394574 & 0.574673881 & 6 & -1.423805324 & 0.060910301 & 1.311879929 \\
\hline 1 & -2.589309770 & 1.204324387 & -1.293994168 & 27 & 0.032143092 & 0.008304737 & -0.051049110 \\
\hline 1 & -2.589309770 & -1.204324387 & -1.293994168 & 6 & 1.749505787 & -0.744368794 & -1.133612933 \\
\hline
\end{tabular}




\begin{tabular}{|c|c|c|c|c|c|c|c|}
\hline 1 & -1.303034543 & 2.315574755 & 1.255255410 & 6 & 1.251028003 & -1.390259962 & 1.028079341 \\
\hline 1 & -1.303034543 & -2.315574755 & 1.255255410 & 6 & 2.261612120 & 0.235067346 & -0.247320580 \\
\hline 27 & -0.236293368 & 0.000000000 & 0.030090689 & 6 & 1.943275797 & -0.142215859 & 1.084269659 \\
\hline 6 & 1.534202649 & 1.146175368 & -0.631639602 & 6 & 1.124911439 & -1.765018016 & -0.338165597 \\
\hline 6 & 1.534202649 & -1.146175368 & -0.631639602 & 1 & -3.451817947 & -1.133723864 & -1.114978944 \\
\hline 6 & 1.623856348 & 0.710123320 & 0.731120018 & 1 & -3.440886634 & -0.677467921 & 1.436314333 \\
\hline 6 & 1.623856348 & -0.710123320 & 0.731120018 & 1 & -1.199366040 & -0.819200124 & -2.266764936 \\
\hline 6 & 1.504636333 & 0.000000000 & -1.463443103 & 1 & -0.980644970 & 1.163009902 & 0.467136272 \\
\hline 1 & 1.475037278 & 2.175272829 & -0.961854915 & 1 & -1.277959103 & 0.310246225 & 2.361218435 \\
\hline 1 & 1.475037278 & -2.175272829 & -0.961854915 & 1 & 1.819862389 & -0.731499801 & -2.212335631 \\
\hline 1 & 1.681581109 & 1.348772391 & 1.602459541 & 1 & 0.858474466 & -1.940957947 & 1.871261191 \\
\hline 1 & 1.681581109 & -1.348772391 & 1.602459541 & 1 & 2.740556811 & 1.158414307 & -0.543355325 \\
\hline 1 & 1.397192627 & 0.000000000 & -2.540942824 & 1 & 2.186191109 & 0.410809823 & 1.981498138 \\
\hline & & & & 1 & 0.650504519 & -2.662346222 & -0.708527984 \\
\hline & & & & 6 & 0.204989122 & 2.995672900 & -0.005988491 \\
\hline & & & & 6 & -0.437233114 & 1.937506245 & -0.517495844 \\
\hline & & & & 1 & 0.184092984 & 3.970534315 & -0.492061963 \\
\hline & & & & 1 & 0.777418075 & 2.933718423 & 0.917154854 \\
\hline & & & & 1 & -1.042696324 & 2.054018361 & -1.412262440 \\
\hline $\mathrm{TS}$ & & & & $\mathrm{TS}$ & & & \\
\hline 6 & -1.373438900 & 0.943784685 & -0.040206775 & 6 & 2.272058419 & -0.955210953 & 0.899574460 \\
\hline 6 & -1.440365158 & -1.016565634 & 0.092479044 & 6 & 2.106116388 & -1.592245654 & -0.316534086 \\
\hline 6 & -0.778050473 & 1.489934228 & 0.966157012 & 6 & 1.226439905 & -0.061236661 & 1.205075682 \\
\hline 6 & -0.720006417 & -1.417796215 & 1.284733504 & 6 & 1.122812106 & -1.037954486 & -1.214167753 \\
\hline 27 & 0.372058288 & 0.018027842 & 0.528647813 & 27 & -0.133042748 & 0.061519877 & -0.010252519 \\
\hline 6 & 2.191938508 & 1.182299338 & -0.101732187 & 6 & -2.150549376 & 0.955280309 & -0.386605035 \\
\hline 6 & 2.076746455 & -1.105582424 & -0.246752700 & 6 & -1.793463875 & -0.804650792 & 1.064525086 \\
\hline 6 & 2.253233926 & 0.655573404 & 1.231662811 & 6 & -2.001915894 & -0.216461340 & -1.157896231 \\
\hline 6 & 2.205121822 & -0.762288221 & 1.139779818 & 6 & -1.777401747 & -1.314321139 & -0.257658196 \\
\hline 6 & 2.105280998 & 0.098734474 & -1.001858791 & 6 & -1.984411678 & 0.611421045 & 0.987938378 \\
\hline 1 & -2.072560895 & 1.154273929 & -0.840411948 & 1 & 3.093404493 & -1.175514262 & 1.579316010 \\
\hline 1 & -1.195128951 & -1.522487813 & -0.841539543 & 1 & 2.659085991 & -2.504430295 & -0.555144783 \\
\hline 1 & -2.511389508 & -0.842308271 & 0.180474370 & 1 & 1.246227887 & 0.450857115 & 2.171001728 \\
\hline 1 & -0.756433274 & 2.434979472 & 1.491936263 & 1 & 1.461959374 & -0.113248237 & -1.684052302 \\
\hline 1 & -0.163967183 & -2.352090666 & 1.251377238 & 1 & 0.725158254 & -1.719634664 & -1.967668712 \\
\hline 1 & -1.172908134 & -1.199435128 & 2.250053980 & 1 & -2.284153329 & 1.955787435 & -0.775840803 \\
\hline 1 & 2.170521649 & 2.232474009 & -0.363920623 & 1 & -1.645690957 & -1.378316209 & 1.969441380 \\
\hline 1 & 2.008652765 & -2.109297484 & -0.647824187 & 1 & -2.035778708 & -0.279117976 & -2.237102420 \\
\hline 1 & 2.340277008 & 1.233491830 & 2.142069500 & 1 & -1.632051813 & -2.347818047 & -0.539185968 \\
\hline 1 & 2.249770712 & -1.456579721 & 1.968105700 & 1 & -2.031343807 & 1.292520534 & 1.827099313 \\
\hline \multirow[t]{5}{*}{1} & 1.996449760 & 0.174274363 & -2.077075301 & 6 & 1.986508377 & 2.162535095 & -0.539955222 \\
\hline & & & & 6 & 0.821638818 & 1.757751319 & -0.017520385 \\
\hline & & & & 1 & 2.290445653 & 3.208998101 & -0.514699639 \\
\hline & & & & 1 & 2.686482071 & 1.485041986 & -1.025726476 \\
\hline & & & & 1 & 0.229735196 & 2.508241899 & 0.512987491 \\
\hline \multicolumn{4}{|c|}{$\mathrm{TS}_{3-5}$} & \multicolumn{4}{|c|}{$\mathrm{TS}_{15-13}$} \\
\hline 6 & 1.580316326 & 1.490463957 & 0.729956793 & 6 & 1.353681106 & -1.309629788 & 1.116990273 \\
\hline 6 & 1.580316326 & 1.490463957 & -0.729956793 & 6 & 0.915412383 & -1.914219602 & -0.076944567 \\
\hline 6 & 1.044378325 & 0.370321204 & 1.308723824 & 6 & 1.705308081 & 0.066766546 & 1.171655670 \\
\hline 6 & 1.044378325 & 0.370321204 & -1.308723824 & 6 & 1.015145845 & -1.304703626 & -1.372853697 \\
\hline
\end{tabular}




\begin{tabular}{|c|c|c|c|c|c|c|c|}
\hline 27 & -0.289146690 & 0.261923768 & 0.000000000 & 27 & 0.146695276 & 0.131025990 & -0.198687007 \\
\hline 1 & 2.031708519 & 2.325470241 & 1.271742684 & 6 & 1.540481276 & 2.020298941 & -0.299438922 \\
\hline 1 & 2.031708519 & 2.325470241 & -1.271742684 & 6 & 2.151957842 & 0.828651709 & 0.024781826 \\
\hline 1 & 0.986792466 & 0.201757370 & 2.382509291 & 1 & 1.118559061 & -1.811044639 & 2.052546521 \\
\hline 1 & 0.986792466 & 0.201757370 & -2.382509291 & 1 & 0.300526875 & -2.805921272 & 0.042443988 \\
\hline 6 & 2.622869792 & -1.390536038 & 0.680058367 & 1 & 1.564006007 & 0.583006486 & 2.119700900 \\
\hline 6 & 2.622869792 & -1.390536038 & -0.680058367 & 1 & 1.966565121 & -0.971026399 & -1.768612978 \\
\hline 1 & 1.964254417 & -2.044896331 & 1.242312379 & 1 & 0.383249691 & -1.748024019 & -2.139583403 \\
\hline 1 & 1.964254417 & -2.044896331 & -1.242312379 & 1 & 1.798525469 & 2.541123710 & -1.216035328 \\
\hline 1 & 3.409315542 & -0.902334608 & 1.246832454 & 1 & 0.957621097 & 2.573113408 & 0.430379268 \\
\hline 1 & 3.409315542 & -0.902334608 & -1.246832454 & 1 & 2.841685071 & 0.383753026 & -0.681386455 \\
\hline 6 & -1.346443101 & -1.494239596 & 0.000000000 & 6 & -1.647438477 & 1.278170343 & -0.453380113 \\
\hline 6 & -1.777022525 & -0.761562911 & 1.157498795 & 6 & -2.121646607 & -0.339485035 & 1.146614586 \\
\hline 6 & -1.777022525 & -0.761562911 & -1.157498795 & 6 & -1.863460168 & 0.033463490 & -1.100614457 \\
\hline 6 & -2.394336215 & 0.429536433 & 0.717022722 & 6 & -2.271895263 & -0.932656874 & -0.085449761 \\
\hline 6 & -2.394336215 & 0.429536433 & -0.717022722 & 6 & -1.628236090 & 1.014015874 & 0.937626098 \\
\hline 1 & -0.851173875 & -2.455710838 & 0.000000000 & 1 & -1.445713747 & 2.224611193 & -0.936203479 \\
\hline 1 & -1.598294955 & -1.045901344 & 2.186026662 & 1 & -2.274328585 & -0.804333193 & 2.113251676 \\
\hline 1 & -1.598294955 & -1.045901344 & -2.186026662 & 1 & -1.949306813 & -0.111397270 & -2.170346886 \\
\hline 1 & -2.773440727 & 1.221481274 & 1.350661371 & 1 & -2.569803379 & -1.955270669 & -0.282369051 \\
\hline 1 & -2.773440727 & 1.221481274 & -1.350661371 & 1 & -1.477689072 & 1.750057671 & 1.718863296 \\
\hline \multicolumn{4}{|c|}{$\mathrm{TS}_{6-7}$} & \multicolumn{4}{|c|}{$\mathrm{TS}_{16-17}$} \\
\hline 6 & -1.834685593 & -1.620527559 & 0.737815078 & 6 & -1.268761000 & 2.472918000 & 1.094713000 \\
\hline 6 & -1.812971154 & -1.683647521 & -0.710530332 & 6 & -1.261835000 & 2.658944000 & -0.347661000 \\
\hline 6 & -0.858231767 & -0.852622439 & 1.278573778 & 6 & -0.922969000 & 1.236491000 & 1.530201000 \\
\hline 6 & -0.967416980 & -0.809098395 & -1.316828053 & 6 & -1.025299000 & 1.533867000 & -1.058077000 \\
\hline 1 & -2.590135544 & -2.151392353 & 1.320558255 & 6 & -2.511750000 & -0.878214000 & -0.852032000 \\
\hline 1 & -2.423310368 & -2.397704708 & -1.266499256 & 6 & -2.504273000 & -1.203373000 & 0.532443000 \\
\hline 1 & -0.737823258 & -0.721998701 & 2.356421710 & 6 & -1.379065000 & -1.518691000 & -1.461540000 \\
\hline 1 & -0.810545233 & -0.783479554 & -2.393593472 & 6 & -1.371940000 & -2.034686000 & 0.786624000 \\
\hline 27 & 0.328480292 & -0.086057424 & -0.000293651 & 6 & -0.698654000 & -2.237658000 & -0.448047000 \\
\hline 6 & 2.170216515 & 0.005649886 & 1.104162339 & 27 & -0.775736000 & -0.041348000 & -0.003663000 \\
\hline 6 & 2.235487729 & -0.061672242 & -1.199654142 & 1 & -1.554015000 & 3.280295000 & 1.770767000 \\
\hline 6 & 1.883722910 & -1.337068473 & 0.693190488 & 1 & -1.418394000 & 3.642767000 & -0.790931000 \\
\hline 6 & 1.902060812 & -1.371745242 & -0.727065809 & 1 & -0.925246000 & 0.941699000 & 2.577577000 \\
\hline 6 & 2.416741658 & 0.771041797 & -0.073005867 & 1 & -0.994054000 & 1.529868000 & -2.148970000 \\
\hline 1 & 2.229659309 & 0.361931322 & 2.124139882 & 1 & -3.245557000 & -0.265139000 & -1.354806000 \\
\hline 1 & 2.319429409 & 0.237148643 & -2.236920711 & 1 & -3.213278000 & -0.848395000 & 1.266872000 \\
\hline 1 & 1.667526709 & -2.171774509 & 1.345289145 & 1 & -1.102342000 & -1.466208000 & -2.505118000 \\
\hline 1 & 1.688916459 & -2.236738186 & -1.340160264 & 1 & -1.083679000 & -2.440819000 & 1.746530000 \\
\hline 1 & 2.641393537 & 1.830261579 & -0.098256351 & 1 & 0.228142000 & -2.779690000 & -0.578448000 \\
\hline 6 & -0.541832552 & 1.708209805 & 0.238783358 & 6 & 1.268404000 & 0.177733000 & 0.154552000 \\
\hline 6 & -1.292311014 & 1.145587303 & -0.828541335 & 6 & 1.954674000 & -0.712527000 & 1.000265000 \\
\hline 1 & -1.009595305 & 1.802588525 & 1.214285614 & 6 & 3.324716000 & -0.934101000 & 0.853936000 \\
\hline 1 & 0.231052670 & 2.440092299 & 0.019126045 & 6 & 4.039592000 & -0.247363000 & -0.129806000 \\
\hline 1 & -2.325806062 & 0.895283585 & -0.621139005 & 6 & 3.379489000 & 0.666725000 & -0.953651000 \\
\hline \multirow[t]{4}{*}{1} & -1.096953178 & 1.495206561 & -1.839715445 & 6 & 2.007485000 & 0.883281000 & -0.810391000 \\
\hline & & & & 1 & 1.410109000 & -1.235126000 & 1.782357000 \\
\hline & & & & 1 & 3.833989000 & -1.634805000 & 1.510325000 \\
\hline & & & & 1 & 5.106890000 & -0.414889000 & -0.245492000 \\
\hline
\end{tabular}




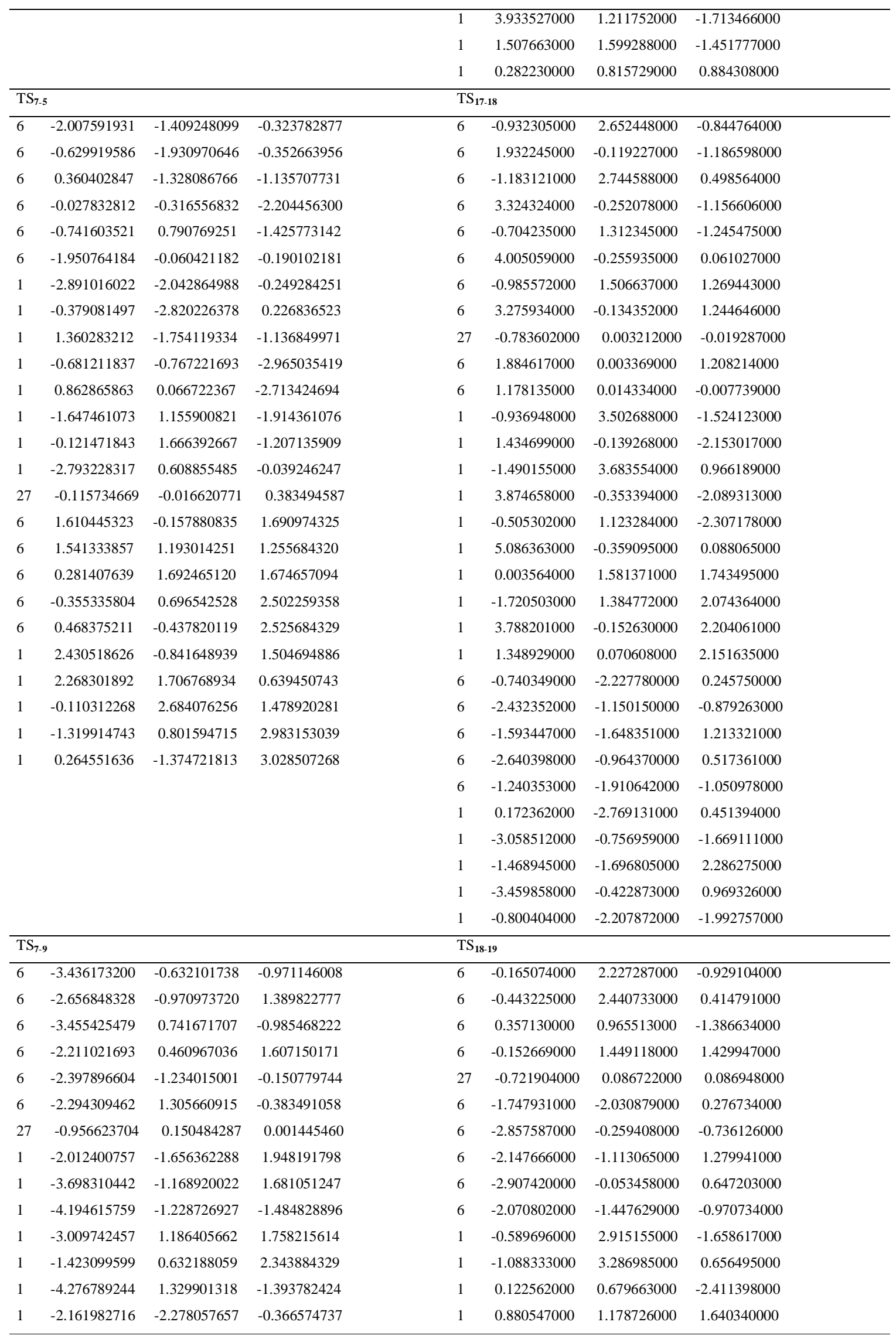




\begin{tabular}{|c|c|c|c|c|c|c|c|}
\hline 1 & -2.217997009 & 2.393065881 & -0.284450358 & 1 & -0.733491000 & 1.553997000 & 2.345970000 \\
\hline 6 & 0.913359798 & -1.026348577 & 0.211908672 & 1 & -1.173977000 & -2.934701000 & 0.432928000 \\
\hline 6 & 0.953720201 & 1.259592521 & -0.056523839 & 1 & -3.297119000 & 0.368559000 & -1.499966000 \\
\hline 6 & 0.579040571 & -0.737132368 & -1.153522066 & 1 & -2.031734000 & -1.244606000 & 2.348131000 \\
\hline 6 & 0.615835054 & 0.674504890 & -1.321253103 & 1 & -3.397273000 & 0.761929000 & 1.163015000 \\
\hline 6 & 1.163195610 & 0.202395556 & 0.867581137 & 1 & -1.868728000 & -1.875255000 & -1.945741000 \\
\hline 1 & 0.971270057 & -2.012142264 & 0.655966479 & 6 & 1.506765000 & -1.151938000 & -0.769496000 \\
\hline 1 & 1.055050958 & 2.318047368 & 0.147377681 & 6 & 2.527067000 & -1.859465000 & -0.116239000 \\
\hline 1 & 0.349406807 & -1.464147014 & -1.921429087 & 6 & 3.543199000 & -1.175878000 & 0.538795000 \\
\hline 1 & 0.411613516 & 1.211885401 & -2.238317691 & 6 & 3.551409000 & 0.230002000 & 0.534120000 \\
\hline 1 & 1.401100879 & 0.321201977 & 1.918221868 & 6 & 2.542169000 & 0.937399000 & -0.098526000 \\
\hline & & & & 6 & 1.487278000 & 0.264386000 & -0.763748000 \\
\hline & & & & 1 & 0.756108000 & -1.689746000 & -1.338099000 \\
\hline & & & & 1 & 2.523473000 & -2.945411000 & -0.138622000 \\
\hline & & & & 1 & 4.338277000 & -1.722265000 & 1.037524000 \\
\hline & & & & 1 & 4.361730000 & 0.766251000 & 1.020230000 \\
\hline & & & & 1 & 2.553208000 & 2.022928000 & -0.105329000 \\
\hline $\mathrm{TS}_{9}$ & & & & $\mathrm{TS}_{3}$ & & & \\
\hline 6 & 1.865055187 & 1.196749965 & 0.692681733 & 6 & 1.929005836 & 1.700125313 & 0.695350496 \\
\hline 6 & 1.865087043 & 1.196898745 & -0.692380572 & 6 & 1.929005836 & 1.700125313 & -0.695350496 \\
\hline 6 & 1.371329119 & 0.001086717 & 1.321960209 & 6 & 1.105026028 & 0.699497972 & 1.241574832 \\
\hline 6 & 1.371381174 & 0.001358734 & -1.321898334 & 6 & 1.105026028 & 0.699497972 & -1.241574832 \\
\hline 6 & -2.268769328 & -1.137290221 & 0.000214719 & 27 & -0.053471953 & -0.161703153 & 0.000000000 \\
\hline 6 & -2.022418979 & -0.333288743 & 1.140233972 & 1 & 2.615768068 & 2.305186624 & 1.290716009 \\
\hline 6 & -2.022471234 & -0.333720497 & -1.140092837 & 1 & 2.615768068 & 2.305186624 & -1.290716009 \\
\hline 6 & -1.798373346 & 1.027162792 & 0.704046550 & 1 & 0.965050860 & 0.606567626 & 2.320890232 \\
\hline 6 & -1.798405805 & 1.026897528 & -0.704383525 & 1 & 0.965050860 & 0.606567626 & -2.320890232 \\
\hline 27 & -0.100241972 & -0.171194139 & -0.000008028 & 6 & 2.186357532 & -1.151616846 & 0.704866983 \\
\hline 6 & 1.879087866 & -1.351351407 & 0.785272239 & 6 & 2.186357532 & -1.151616846 & -0.704866983 \\
\hline 6 & 1.879240769 & -1.351166859 & -0.785524664 & 1 & 1.583890597 & -1.888680704 & 1.232229165 \\
\hline 1 & 2.079218954 & 2.100531250 & 1.261048781 & 1 & 1.583890597 & -1.888680704 & -1.232229165 \\
\hline 1 & 2.079186557 & 2.100832467 & -1.260529907 & 1 & 3.058494285 & -0.813858593 & 1.252391019 \\
\hline 1 & 1.193457668 & 0.029306605 & 2.396165965 & 1 & 3.058494285 & -0.813858593 & -1.252391019 \\
\hline 1 & 1.193476281 & 0.029797493 & -2.396120466 & 6 & -1.553474822 & -1.828550240 & 0.000000000 \\
\hline 1 & -2.474506847 & -2.201138320 & 0.000417074 & 6 & -1.781663838 & -1.019794535 & 1.147643052 \\
\hline 1 & -2.093954602 & -0.651768968 & 2.173809626 & 6 & -1.781663838 & -1.019794535 & -1.147643052 \\
\hline 1 & -2.094069168 & -0.652580280 & -2.173553950 & 6 & -2.230475018 & 0.266991691 & 0.704388952 \\
\hline 1 & -1.650784049 & 1.882105722 & 1.351729314 & 6 & -2.230475018 & 0.266991691 & -0.704388952 \\
\hline 1 & -1.650793911 & 1.881589234 & -1.352393373 & 1 & -1.232287346 & -2.863144467 & 0.000000000 \\
\hline 1 & 1.256434515 & -2.164268597 & 1.181065074 & 1 & -1.684168353 & -1.334166193 & 2.179658188 \\
\hline 1 & 1.256744951 & -2.164054636 & -1.181645147 & 1 & -1.684168353 & -1.334166193 & -2.179658188 \\
\hline 1 & 2.892804129 & -1.540183728 & 1.162732523 & 1 & -2.474509804 & 1.106232489 & 1.343216410 \\
\hline 1 & 2.893066201 & -1.539799074 & -1.162846976 & 1 & -2.474509804 & 1.106232489 & -1.343216410 \\
\hline \multicolumn{4}{|c|}{$\mathrm{TS}_{8-7}$} & \multicolumn{4}{|c|}{$\mathrm{TS}_{20-20}$} \\
\hline 6 & 2.427153964 & 0.309334176 & 0.450542913 & 6 & 1.620899929 & -0.157973542 & -1.402823827 \\
\hline 6 & 2.250477446 & -1.009062050 & 1.144162062 & 6 & 2.428337518 & 0.969221280 & -0.780674147 \\
\hline 6 & 1.430243155 & 0.810909065 & -0.294352907 & 6 & 1.339636078 & -1.329328264 & -0.728753903 \\
\hline 6 & 0.905625366 & -1.625653032 & 0.723177472 & 27 & -0.196301185 & -0.029899827 & 0.000369716 \\
\hline 27 & -0.273139994 & -0.180554028 & 0.006880885 & 6 & 2.428453462 & 0.969023867 & 0.780399220 \\
\hline 6 & -1.139173522 & 1.437515697 & 1.259753138 & 6 & 1.338906725 & -1.329146877 & 0.728424155 \\
\hline
\end{tabular}




\begin{tabular}{|c|c|c|c|c|c|c|c|}
\hline 6 & -2.001148883 & -0.690476220 & 1.130820666 & 6 & 1.619486496 & -0.157333497 & 1.402282408 \\
\hline 6 & -1.970136320 & 1.366157711 & 0.097749563 & 1 & 1.492822094 & -0.126815078 & -2.481980765 \\
\hline 6 & -2.514109220 & 0.064957363 & 0.034990061 & 1 & 2.063614926 & 1.931286638 & -1.160588853 \\
\hline 6 & -1.176845936 & 0.183976833 & 1.914511511 & 1 & 3.464408880 & 0.886191172 & -1.143033784 \\
\hline 1 & 3.327920181 & 0.898661765 & 0.644072142 & 1 & 0.979040998 & -2.206051078 & -1.259013023 \\
\hline 1 & 2.275927799 & -0.826162656 & 2.231729314 & 1 & 2.065056927 & 1.931456951 & 1.160582105 \\
\hline 1 & 3.095777912 & -1.688158039 & 0.944719518 & 1 & 3.464425317 & 0.884430958 & 1.142602689 \\
\hline 1 & 1.497417419 & 1.813427231 & -0.715066886 & 1 & 0.978291561 & -2.205854404 & 1.258646807 \\
\hline 1 & 1.044729164 & -2.359221046 & -0.073977419 & 1 & 1.491141327 & -0.125968952 & 2.481384840 \\
\hline 1 & 0.395872923 & -2.121445306 & 1.554393910 & 6 & -2.056260579 & 0.617308071 & 1.157268245 \\
\hline 1 & -0.560158362 & 2.296809490 & 1.572478854 & 6 & -2.357464703 & -0.696093241 & 0.713112488 \\
\hline 1 & -2.238384566 & -1.720469885 & 1.362998888 & 6 & -1.843462196 & 1.429834484 & 0.000259159 \\
\hline 1 & -2.121473190 & 2.158502743 & -0.624478439 & 6 & -2.357081643 & -0.696151122 & -0.712718048 \\
\hline 1 & -3.149791370 & -0.320220753 & -0.752529393 & 6 & -2.055673410 & 0.617264142 & -1.156813103 \\
\hline 1 & -0.649875179 & -0.078824567 & 2.821830981 & 1 & -1.996755197 & 0.948753902 & 2.186630002 \\
\hline 6 & 0.590778809 & -0.098520059 & -1.867776905 & 1 & -2.558763390 & -1.551117310 & 1.347767441 \\
\hline 6 & -0.228686476 & -1.054101514 & -1.708898599 & 1 & -1.635770836 & 2.493908132 & 0.000277560 \\
\hline 1 & 1.137510119 & 0.511337701 & -2.568423691 & 1 & -2.558198066 & -1.551185916 & -1.347442238 \\
\hline 1 & -0.743743238 & -1.890780619 & -2.150104641 & 1 & -1.995947961 & 0.948688972 & -2.186165145 \\
\hline \multicolumn{4}{|c|}{$\mathrm{TS}_{7-\mathbf{1 1}}$} & \multicolumn{4}{|c|}{$\mathrm{TS}_{20-21}$} \\
\hline 6 & -1.913432090 & -0.692092862 & 0.986906999 & 6 & 3.393752680 & -1.069247665 & 1.673843510 \\
\hline 6 & -2.284905104 & -1.067972168 & -0.371764384 & 6 & 2.896857398 & -1.559368449 & 0.312374640 \\
\hline 6 & -1.527983987 & -1.457374334 & -1.419994107 & 6 & 3.857654490 & -2.526403264 & -0.442038340 \\
\hline 6 & -0.691684938 & -0.536224265 & 1.567313412 & 6 & 5.248354550 & -2.642816827 & 0.134341448 \\
\hline 1 & -2.785965487 & -0.430353757 & 1.595515266 & 6 & 5.576341268 & -2.212971388 & 1.368050397 \\
\hline 1 & -3.351803071 & -0.964917006 & -0.573426428 & 6 & 4.619890457 & -1.481625474 & 2.213735978 \\
\hline 1 & -2.051387581 & -1.583168122 & -2.369071418 & 27 & 4.872072212 & 0.341684347 & 1.364699435 \\
\hline 1 & -0.737675619 & -0.150848302 & 2.593920446 & 6 & 4.486048137 & 2.430123822 & 2.406775841 \\
\hline 6 & -0.052629669 & -1.730693812 & -1.486951940 & 6 & 4.501552243 & 2.515457825 & 0.986461398 \\
\hline 1 & 0.439730142 & -0.851078926 & -1.945833802 & 6 & 5.803758333 & 2.126835075 & 0.533201837 \\
\hline 1 & 0.087925282 & -2.532295657 & -2.232588000 & 6 & 6.578100426 & 1.793748640 & 1.686891752 \\
\hline 6 & 0.645518456 & -2.143713207 & -0.193525561 & 6 & 5.763737045 & 1.985483334 & 2.837842235 \\
\hline 1 & 1.621544478 & -2.586641626 & -0.429087965 & 1 & 2.625736984 & -0.693851488 & 2.346593903 \\
\hline 1 & 0.050171697 & -2.893754057 & 0.336489260 & 1 & 1.935467185 & -2.066712453 & 0.472085495 \\
\hline 27 & 1.083414173 & -0.661639735 & 0.997544742 & 1 & 2.657249882 & -0.699045847 & -0.327441655 \\
\hline 6 & 2.007537473 & 1.112049223 & 1.876962189 & 1 & 3.422293657 & -3.537638247 & -0.463967424 \\
\hline 6 & 2.436507364 & -0.030536966 & 2.571507701 & 1 & 3.928372250 & -2.228990262 & -1.496467186 \\
\hline 6 & 3.118990828 & -0.901812997 & 1.640317069 & 1 & 5.990115302 & -3.156858232 & -0.474529237 \\
\hline 6 & 3.121542598 & -0.279347503 & 0.377220378 & 1 & 6.579590623 & -2.373565566 & 1.756477465 \\
\hline 6 & 2.355600353 & 0.921690351 & 0.497849377 & 1 & 4.807029965 & -1.423961721 & 3.283035626 \\
\hline 1 & 1.415826301 & 1.925391210 & 2.274424129 & 1 & 3.638397006 & 2.649912306 & 3.044636409 \\
\hline 1 & 2.250152943 & -0.254893223 & 3.614307485 & 1 & 3.685540722 & 2.856013571 & 0.360007479 \\
\hline 1 & 3.539251403 & -1.869918648 & 1.881229889 & 1 & 6.159324541 & 2.149844134 & -0.489840039 \\
\hline 1 & 3.514393706 & -0.690295850 & -0.542920065 & 1 & 7.614605610 & 1.477589084 & 1.685225852 \\
\hline 1 & 2.110044347 & 1.597836241 & -0.311717672 & 1 & 6.064676403 & 1.811088653 & 3.863877641 \\
\hline \multicolumn{4}{|c|}{$\mathrm{TS}_{11-12}$} & \multicolumn{4}{|c|}{$\mathrm{TS}_{21-21}$} \\
\hline 6 & -1.899339862 & -0.621792175 & 0.939874415 & 6 & 1.622218845 & -0.156932420 & -1.402536047 \\
\hline 6 & -2.384365219 & -1.265878494 & -0.273183334 & 6 & 2.430428779 & 0.969004913 & -0.780226712 \\
\hline 6 & -1.629653911 & -1.587532144 & -1.343375213 & 6 & 1.338871581 & -1.327565044 & -0.728573863 \\
\hline 6 & -0.153162004 & -1.474467640 & -1.393511938 & 27 & -0.196727623 & -0.028011238 & -0.000002044 \\
\hline
\end{tabular}




\begin{tabular}{|c|c|c|c|c|c|c|c|}
\hline 6 & 0.682345736 & -2.074066449 & -0.410684856 & 6 & 2.430499525 & 0.968954570 & 0.780177660 \\
\hline 27 & 0.816614584 & -0.105508846 & -0.073625298 & 6 & 1.338614119 & -1.327491203 & 0.728552738 \\
\hline 6 & -0.660954345 & -0.110280695 & 1.122735566 & 6 & 1.622008430 & -0.156821441 & 1.402468006 \\
\hline 6 & 1.649564638 & 1.740298326 & 0.588633840 & 1 & 1.495108758 & -0.126418360 & -2.481118190 \\
\hline 6 & 2.229592111 & 0.653905200 & 1.320881764 & 1 & 2.067760843 & 1.930691121 & -1.160930256 \\
\hline 6 & 2.937596276 & -0.161270198 & 0.400019362 & 1 & 3.465447383 & 0.883744113 & -1.142502142 \\
\hline 6 & 2.804076295 & 0.413342174 & -0.900643587 & 1 & 0.978439410 & -2.203176689 & -1.259232664 \\
\hline 6 & 2.022661443 & 1.592954194 & -0.777024947 & 1 & 2.067940211 & 1.930669692 & 1.160905035 \\
\hline 1 & -2.640470229 & -0.502591970 & 1.733970395 & 1 & 3.465535615 & 0.883559381 & 1.142390346 \\
\hline 1 & -3.455227396 & -1.457909514 & -0.329810416 & 1 & 0.977866875 & -2.203012455 & 1.259163410 \\
\hline 1 & -2.101533392 & -1.972805989 & -2.245539410 & 1 & 1.494783624 & -0.126222883 & 2.481043614 \\
\hline 1 & 0.268996378 & -1.369008750 & -2.393009604 & 6 & -2.057076871 & 0.616419813 & 1.157034870 \\
\hline 1 & 1.663800870 & -2.429126383 & -0.712431924 & 6 & -2.360262161 & -0.696237119 & 0.712730199 \\
\hline 1 & 0.228495176 & -2.588075467 & 0.430403807 & 6 & -1.843298729 & 1.428970524 & 0.000062378 \\
\hline 1 & -0.476723665 & 0.378412799 & 2.082882513 & 6 & -2.360211398 & -0.696243282 & -0.712682630 \\
\hline 1 & 1.033806280 & 2.529539589 & 0.998317748 & 6 & -2.057084401 & 0.616458816 & -1.156929479 \\
\hline 1 & 2.132401110 & 0.476843896 & 2.383650034 & 1 & -1.998195154 & 0.946595511 & 2.185872852 \\
\hline 1 & 3.469641582 & -1.072037301 & 0.642012342 & 1 & -2.562948834 & -1.550717801 & 1.346153932 \\
\hline 1 & 3.216839905 & 0.015578432 & -1.819071023 & 1 & -1.635752662 & 2.492241365 & 0.000073385 \\
\hline 1 & 1.719542611 & 2.242882644 & -1.587873586 & 1 & -2.562867163 & -1.550693238 & -1.346128110 \\
\hline 1 & -0.210427971 & 0.197661763 & -1.101416653 & 1 & -1.998255934 & 0.946682813 & -2.185766287 \\
\hline \multicolumn{4}{|c|}{$\mathrm{TS}_{12-13}$} & \multicolumn{4}{|c|}{$\mathrm{TS}_{7-21}$} \\
\hline 6 & -2.184278482 & -0.767379862 & 0.866145181 & 6 & -2.453836237 & 0.786778755 & -0.891727545 \\
\hline 6 & -2.695364921 & -1.196173918 & -0.424811213 & 6 & -2.909474194 & -0.602102504 & -0.956357600 \\
\hline 6 & -1.937162367 & -1.450694188 & -1.516264574 & 6 & -2.145884134 & -1.676417505 & -1.234863248 \\
\hline 6 & -0.475985080 & -1.619647056 & -1.523360559 & 6 & -0.656385843 & -1.680578336 & -1.488027284 \\
\hline 6 & 0.208237321 & -2.372129800 & -0.563212221 & 6 & 0.006020430 & -0.302742463 & -1.621263532 \\
\hline 27 & 0.570413849 & -0.399434092 & -0.104955410 & 27 & 0.011019797 & 0.277875948 & 0.368866523 \\
\hline 6 & -0.915754753 & -0.341512196 & 1.081888102 & 6 & -1.165510909 & 1.194242083 & -0.779430715 \\
\hline 6 & 1.693564579 & 1.256007878 & 0.570479608 & 1 & -3.226254704 & 1.548199213 & -1.017081221 \\
\hline 6 & 2.130849221 & 0.106589048 & 1.295813145 & 1 & -3.978537523 & -0.759692069 & -0.818968139 \\
\hline 6 & 2.650265180 & -0.818685519 & 0.360748087 & 1 & -2.624756251 & -2.654019792 & -1.273745091 \\
\hline 6 & 2.592043631 & -0.217028633 & -0.945020386 & 1 & -0.458447933 & -2.253361301 & -2.409835083 \\
\hline 6 & 2.025639668 & 1.066656306 & -0.812585395 & 1 & -0.165517907 & -2.264734653 & -0.690066599 \\
\hline 1 & -2.913597026 & -0.687683761 & 1.674407941 & 1 & -0.292914118 & 0.196171563 & -2.542971305 \\
\hline 1 & -3.778666969 & -1.220060117 & -0.538146739 & 1 & 1.098453244 & -0.374398717 & -1.639148993 \\
\hline 1 & -2.427275758 & -1.590861881 & -2.479310429 & 1 & -0.889222182 & 2.227001280 & -0.995042823 \\
\hline 1 & 0.016664945 & -1.449074512 & -2.480036888 & 6 & 1.807186536 & -0.504989691 & 1.543138966 \\
\hline 1 & 1.171103771 & -2.806737053 & -0.811913714 & 6 & 2.301260483 & 0.689801822 & 0.953880609 \\
\hline 1 & -0.331404645 & -2.839358051 & 0.253330328 & 6 & 1.532053736 & 1.778955538 & 1.442961017 \\
\hline 1 & -0.549251204 & 0.452707434 & -0.500037165 & 6 & 0.558575719 & 1.262374083 & 2.341049059 \\
\hline 1 & -0.689263155 & 0.097868441 & 2.055671228 & 6 & 0.722862271 & -0.155941957 & 2.406880964 \\
\hline 1 & 1.223697736 & 2.134387944 & 0.993229265 & 1 & 2.201321239 & -1.503020314 & 1.389793081 \\
\hline 1 & 2.017410669 & -0.057528453 & 2.359265217 & 1 & 3.113846720 & 0.755917983 & 0.239616434 \\
\hline 1 & 3.044480759 & -1.799932955 & 0.589856025 & 1 & 1.655329677 & 2.820171157 & 1.170149232 \\
\hline 1 & 2.906450635 & -0.683438224 & -1.870428193 & 1 & -0.176810350 & 1.841383741 & 2.886827137 \\
\hline 1 & 1.819743397 & 1.763901221 & -1.613894242 & 1 & 0.170915433 & -0.833271865 & 3.047565159 \\
\hline \multicolumn{4}{|c|}{$\mathrm{TS}_{6-12}$} & \multicolumn{4}{|c|}{ CP1 } \\
\hline 6 & -2.003974597 & -1.748718297 & -0.244621114 & 27 & -0.077304000 & -0.005488000 & -0.130866000 \\
\hline 6 & -2.230613166 & -0.889930094 & 0.892425225 & 6 & -1.389552999 & 1.315885999 & 0.225333000 \\
\hline
\end{tabular}




\begin{tabular}{|c|c|c|c|c|c|c|c|}
\hline 6 & -0.872793613 & -1.463918481 & -0.939792231 & 6 & -2.559352999 & 0.746654000 & -0.097296000 \\
\hline 6 & -1.426302881 & 0.223215026 & 0.961048131 & 6 & -2.551274999 & -0.752351000 & -0.110283000 \\
\hline 27 & 0.151208538 & -0.035365685 & -0.181807564 & 6 & -1.383504999 & -1.323675999 & 0.221435000 \\
\hline 6 & 1.742923589 & -1.282625215 & 0.335247464 & 1 & -1.233350999 & 2.388267999 & 0.330965000 \\
\hline 6 & 1.848172060 & 0.972847895 & 0.809202230 & 1 & -3.481016998 & 1.292420999 & -0.309652000 \\
\hline 6 & 2.103973562 & -0.586060558 & -0.870488443 & 1 & -3.462368998 & -1.304334999 & -0.351740000 \\
\hline 6 & 2.183785860 & 0.795979207 & -0.561434903 & 1 & -1.225635999 & -2.397132999 & 0.313974000 \\
\hline 6 & 1.595726688 & -0.316732312 & 1.368383018 & 6 & 1.593184999 & 1.185194999 & 0.473430000 \\
\hline 1 & -2.701073021 & -2.545908704 & -0.506666666 & 6 & 1.245860999 & 0.055445000 & 1.311165999 \\
\hline 1 & -2.994058636 & -1.106055325 & 1.639885180 & 6 & 1.945807999 & 0.687840000 & -0.782310000 \\
\hline 1 & -0.569081940 & -2.038574815 & -1.816465354 & 1 & 1.476942999 & 2.225247999 & 0.743198000 \\
\hline 1 & -1.423809224 & 0.877687240 & 1.827233770 & 6 & 1.568421999 & -1.144987999 & 0.567730000 \\
\hline 1 & -0.552662303 & 0.940579589 & -1.196411243 & 1 & 0.955922000 & 0.100182000 & 2.350900999 \\
\hline 1 & 1.610930745 & -2.349982720 & 0.438904434 & 6 & 1.930137999 & -0.759600000 & -0.725798000 \\
\hline 1 & 1.799519353 & 1.915309174 & 1.337427397 & 1 & 2.167332999 & 1.278794999 & -1.661501999 \\
\hline 1 & 2.285532596 & -1.037534087 & -1.835870995 & 1 & 1.429835999 & -2.156394999 & 0.922283000 \\
\hline 1 & 2.394043059 & 1.588764835 & -1.268075034 & 1 & 2.143584999 & -1.423119999 & -1.553371999 \\
\hline 1 & 1.304841869 & -0.523453819 & 2.388713860 & & & & \\
\hline 6 & -1.451537063 & 2.655567008 & -0.329500530 & & & & \\
\hline 6 & -1.491081106 & 1.285965186 & -0.511969262 & & & & \\
\hline 1 & -2.317541509 & 3.266187881 & -0.561449467 & & & & \\
\hline 1 & -0.589982621 & 3.153262409 & 0.102283856 & & & & \\
\hline 1 & -2.343184240 & 0.878386660 & -1.051780759 & & & & \\
\hline \multicolumn{4}{|l|}{ CP2 } & \multicolumn{4}{|c|}{ CP3 } \\
\hline 6 & -2.579936099 & -0.528460300 & 0.786541000 & 27 & 0.521015000 & 0.326140000 & -0.154400000 \\
\hline 6 & -0.558872500 & 2.420861199 & -0.701902200 & 6 & -2.055609999 & 0.607142000 & -0.670273000 \\
\hline 6 & -2.590474399 & -0.580747800 & -0.668211000 & 6 & -1.813486999 & -0.795316000 & -0.268300000 \\
\hline 6 & -0.325586900 & 2.502251399 & 0.624290900 & 6 & -0.927649000 & -1.600883999 & -0.911926000 \\
\hline 6 & -1.365368999 & -0.259457100 & 1.320793499 & 6 & -0.247860000 & -1.239794999 & -2.228377999 \\
\hline 6 & -1.385267599 & -0.344423700 & -1.238226099 & 6 & 0.930891000 & -0.299087000 & -1.966047999 \\
\hline 6 & 1.296460199 & -1.680928999 & -0.543211100 & 6 & -0.948109000 & 1.353474999 & -0.819667000 \\
\hline 6 & 1.377964699 & -1.509409699 & 0.860986600 & 1 & -3.081691999 & 0.970869000 & -0.764547000 \\
\hline 6 & 1.836482399 & -0.508034600 & -1.162260699 & 1 & -2.338071999 & -1.188170999 & 0.604608000 \\
\hline 6 & 1.962189799 & -0.230284500 & 1.117954700 & 1 & -0.735563000 & -2.589431999 & -0.498274000 \\
\hline 6 & 2.275037299 & 0.365154800 & -0.134148200 & 1 & -0.984449000 & -0.737347000 & -2.864186999 \\
\hline 27 & 0.023696700 & 0.061206500 & 0.019858400 & 1 & 0.061815000 & -2.161160999 & -2.737348999 \\
\hline 1 & 0.242478900 & 2.511947499 & -1.429669599 & 1 & 1.006605000 & 0.520047000 & -2.684210999 \\
\hline 1 & -1.564230499 & 2.323590999 & -1.092214900 & 1 & 1.886272999 & -0.827385000 & -1.901293999 \\
\hline 1 & -3.482662798 & -0.707380900 & 1.374657599 & 1 & -0.964402000 & 2.401229999 & -1.119867999 \\
\hline 1 & -1.138178499 & 2.474653999 & 1.340498199 & 6 & 1.816503999 & -0.480822000 & 1.542748999 \\
\hline 1 & 0.673424400 & 2.658799399 & 1.019872800 & 6 & 2.631761999 & 0.221871000 & 0.624714000 \\
\hline 1 & -3.499559398 & -0.804931100 & -1.230184499 & 6 & 2.175258999 & 1.573106999 & 0.567589000 \\
\hline 1 & -1.182114699 & -0.233758100 & 2.397112799 & 6 & 1.098004999 & 1.705556999 & 1.496835999 \\
\hline 1 & -1.220954499 & -0.388033400 & -2.316833299 & 6 & 0.866329000 & 0.438372000 & 2.086615999 \\
\hline 1 & 0.868614700 & -2.531247099 & -1.055384900 & 1 & 1.896492999 & -1.531525999 & 1.789381999 \\
\hline 1 & 1.021430600 & -2.206500299 & 1.606411399 & 1 & 3.432187998 & -0.200241000 & 0.032177000 \\
\hline 1 & 1.918986599 & -0.335046800 & -2.226982099 & 1 & 2.591383999 & 2.361673999 & -0.044407000 \\
\hline 1 & 2.164607699 & 0.189669100 & 2.094356599 & 1 & 0.530508000 & 2.607237999 & 1.683237999 \\
\hline 1 & 2.722985599 & 1.340065499 & -0.279133100 & 1 & 0.101654000 & 0.205717000 & 2.815864999 \\
\hline
\end{tabular}




\begin{tabular}{|c|c|c|c|c|c|c|c|}
\hline 27 & 0.767683000 & -0.063027000 & 0.078660000 & 6 & 1.500696599 & 0.021914700 & -1.366450999 \\
\hline 6 & -2.039261999 & -0.574916000 & 0.926249000 & 6 & 2.397312199 & 1.039885800 & -0.682165300 \\
\hline 6 & -2.527128999 & -1.298829999 & -0.242765000 & 6 & 1.305223599 & -1.254027799 & -0.828115600 \\
\hline 6 & -1.824831999 & -1.665689999 & -1.333097999 & 27 & -0.196928500 & -0.105366000 & -0.015213200 \\
\hline 6 & -0.359760000 & -1.400687999 & -1.519070999 & 6 & 2.339569799 & 0.952249600 & 0.869216500 \\
\hline 6 & 0.547884000 & -1.908888999 & -0.399232000 & 6 & 1.330310499 & -1.381622599 & 0.612486300 \\
\hline 6 & -0.791837000 & -0.102386000 & 1.172808999 & 6 & 1.526409899 & -0.228409000 & 1.368935099 \\
\hline 1 & -2.810839999 & -0.385280000 & 1.677662999 & 1 & 1.334428399 & 0.160523100 & -2.431582399 \\
\hline 1 & -3.585641998 & -1.553952999 & -0.225038000 & 1 & 2.127166999 & 2.047266399 & -1.015405100 \\
\hline 1 & -2.339013999 & -2.173292999 & -2.145936999 & 1 & 3.431923898 & 0.879488600 & -1.021077400 \\
\hline 1 & -0.261890000 & -0.265927000 & -1.593635999 & 1 & 0.992809000 & -2.093961599 & -1.440597299 \\
\hline 1 & -0.004307000 & -1.724788999 & -2.503273999 & 1 & 1.927336199 & 1.876627099 & 1.289174899 \\
\hline 1 & 1.488375999 & -2.335756999 & -0.751527000 & 1 & 3.359615198 & 0.873461000 & 1.274310799 \\
\hline 1 & 0.056630000 & -2.552023999 & 0.326441000 & 1 & 1.024658700 & -2.313428699 & 1.078437400 \\
\hline 1 & -0.664594000 & 0.400702000 & 2.134667999 & 1 & 1.367222599 & -0.283117200 & 2.442881499 \\
\hline 6 & 1.676639999 & 1.806926999 & 0.857630000 & 6 & -1.886970699 & 0.724864400 & 1.099059500 \\
\hline 6 & 2.284319999 & 0.667775000 & 1.470993999 & 6 & -2.245371199 & -0.630148400 & 0.840417500 \\
\hline 6 & 2.949069999 & -0.073981000 & 0.453446000 & 6 & -1.758736299 & 1.392532499 & -0.158208400 \\
\hline 6 & 2.727751999 & 0.582835000 & -0.787317000 & 6 & -2.349107399 & -0.799680600 & -0.567306100 \\
\hline 6 & 1.949518999 & 1.751615999 & -0.531791000 & 6 & -2.048713399 & 0.443552000 & -1.184719699 \\
\hline 1 & 1.083127999 & 2.558148999 & 1.360503999 & 1 & -1.755754899 & 1.172239099 & 2.075069499 \\
\hline 1 & 2.249527999 & 0.414931000 & 2.521554999 & 1 & -2.408030099 & -1.396383899 & 1.587148599 \\
\hline 1 & 3.492407998 & -0.997598000 & 0.598095000 & 1 & -1.527308499 & 2.439358599 & -0.304804600 \\
\hline 1 & 3.092657999 & 0.259498000 & -1.753126999 & 1 & -2.595289499 & -1.719666199 & -1.081247600 \\
\hline 1 & 1.602817999 & 2.458748999 & -1.274095999 & 1 & -2.031143699 & 0.637836300 & -2.249077099 \\
\hline
\end{tabular}

Imaginary Frequencies of Some Species Involved

\begin{tabular}{cc}
\hline $\begin{array}{c}\text { Transition } \\
\text { State }\end{array}$ & Frequency \\
\hline $\mathrm{TS}_{\mathbf{1 - 3}}$ & 426.37 \\
$\mathrm{TS}_{\mathbf{2 - 4}}$ & 447.31 \\
$\mathrm{TS}_{\mathbf{3 - 5}}$ & 315.41 \\
$\mathrm{TS}_{\mathbf{6 - 7}}$ & 309.83 \\
$\mathrm{TS}_{\mathbf{7 - 5}}$ & 462.59 \\
$\mathrm{TS}_{\mathbf{7 - 9}}$ & 223.53 \\
$\mathrm{TS}_{\mathbf{9 - 5}}$ & 336.53 \\
$\mathrm{TS}_{\mathbf{8 - 7}}$ & 355.30 \\
$\mathrm{TS}_{\mathbf{7 - 1 1}}$ & 214.84 \\
$\mathrm{TS}_{\mathbf{1 1 - 1 2}}$ & 584.58 \\
$\mathrm{TS}_{\mathbf{1 2 - 1 3}}$ & 474.48 \\
$\mathrm{TS}_{\mathbf{6 - 1 2}}$ & 336.36
\end{tabular}




$\begin{array}{cc}\mathrm{TS}_{\mathbf{6 - 1 4}} & 990.24 \\ \mathrm{TS}_{\mathbf{1 4 - 1 5}} & 280.18 \\ \mathrm{TS}_{\mathbf{1 5 - 1 3}} & 170.31 \\ \mathrm{TS}_{\mathbf{1 6 - 1 7}} & 1043.93 \\ \mathrm{TS}_{\mathbf{1 7 - 1 8}} & 148.11 \\ \mathrm{TS}_{\mathbf{1 8 - 1 9}} & 450.07 \\ \mathrm{TS}_{\mathbf{3 - 2 0}} & 460.75 \\ \mathrm{TS}_{\mathbf{2 0 - 2 0}} & 102.35 \\ \mathrm{TS}_{\mathbf{2 0 - 2 1}} & 128.79 \\ \mathrm{TS}_{\mathbf{2 1 - 2 1}} & 144.21 \\ \mathrm{TS}_{\mathbf{7 - 2 1}} & 371.66\end{array}$

\section{Species Incorporating Furan and Thiophene}

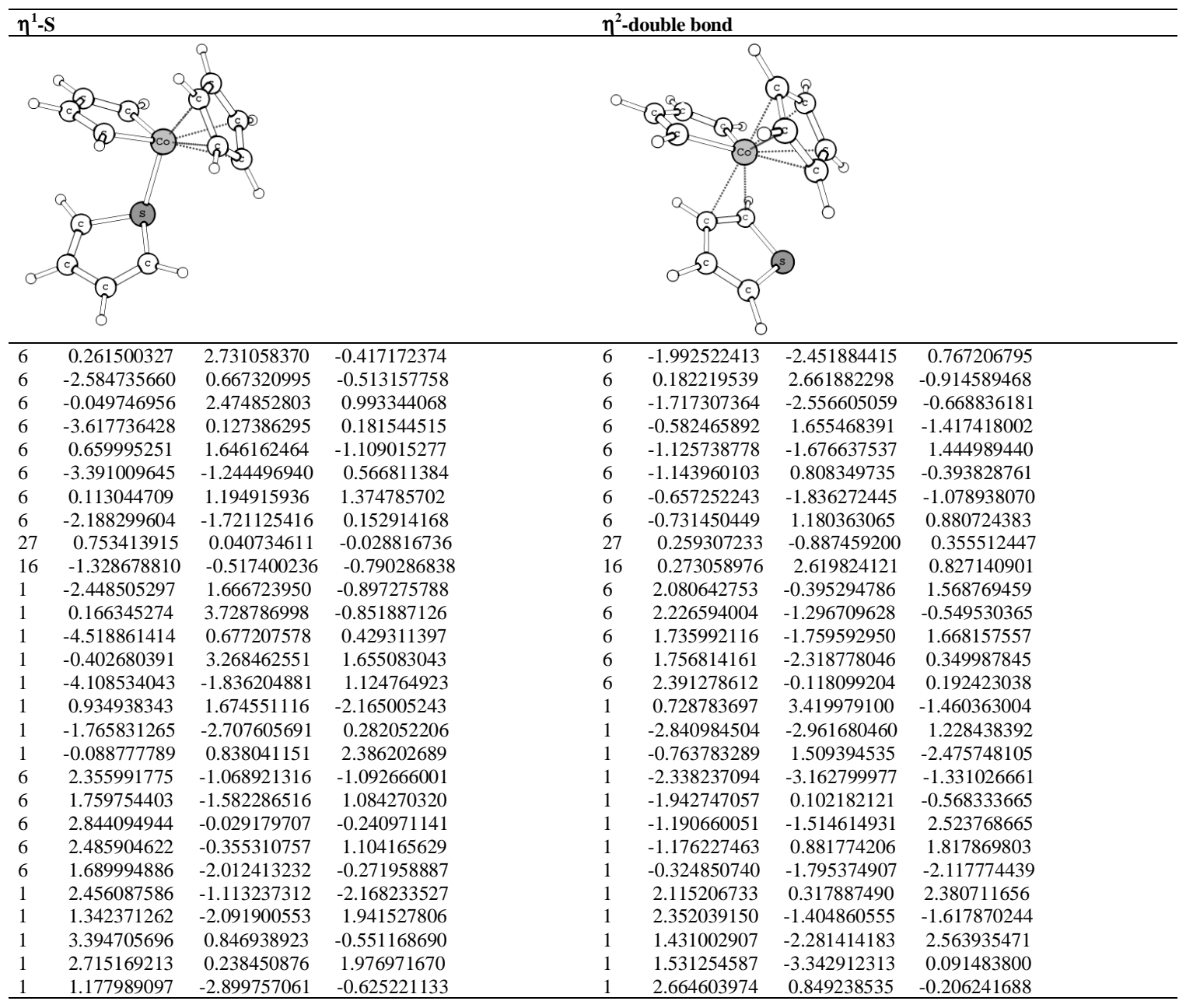




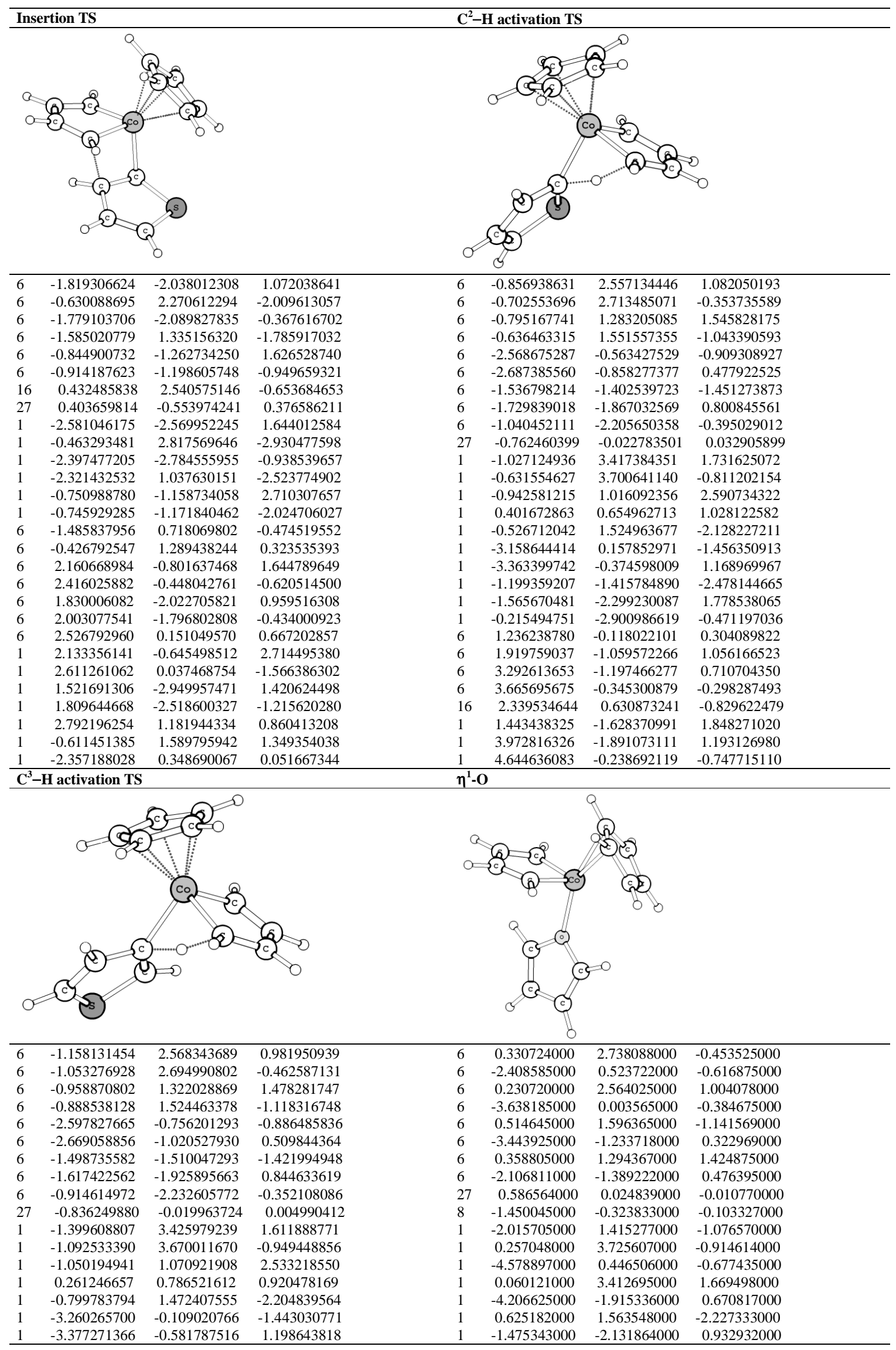




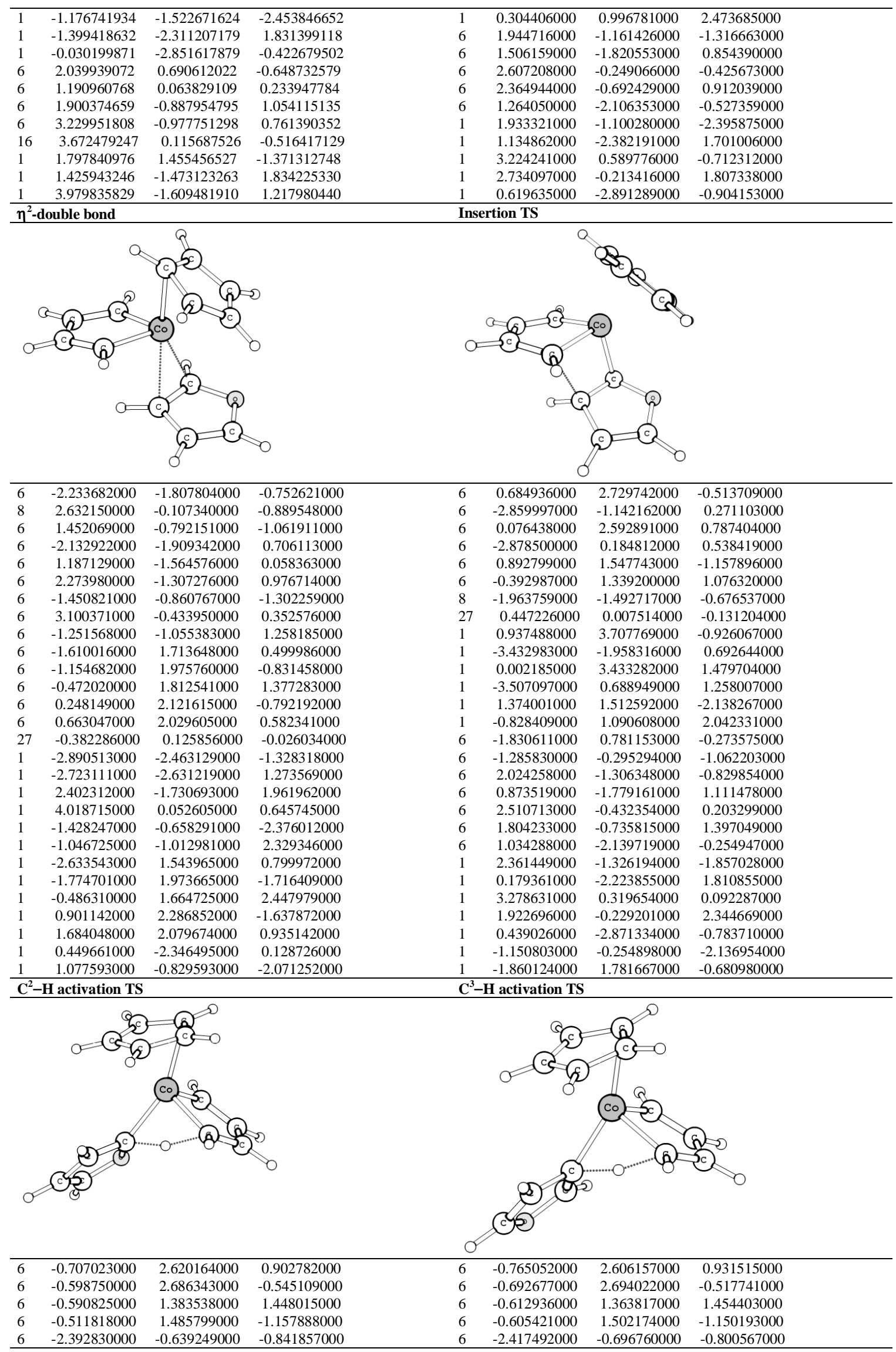




\begin{tabular}{lccccccc}
\hline 6 & -2.474619000 & -0.840320000 & 0.563991000 & 6 & -2.448680000 & -0.920791000 & 0.604027000 \\
6 & -1.362288000 & -1.499959000 & -1.350733000 & 6 & -1.370041000 & -1.509241000 & -1.353192000 \\
6 & -1.498490000 & -1.816552000 & 0.930855000 & 6 & -1.421482000 & -1.858588000 & 0.927379000 \\
6 & -0.833676000 & -2.227550000 & -0.255258000 & 6 & -0.776506000 & -2.226343000 & -0.284593000 \\
27 & -0.576127000 & -0.018379000 & 0.018976000 & 27 & -0.597573000 & -0.017675000 & 0.009193000 \\
1 & -0.883393000 & 3.516540000 & 1.500008000 & 1 & -0.947401000 & 3.489751000 & 1.545162000 \\
1 & -0.561243000 & 3.643002000 & -1.066554000 & 1 & -0.697603000 & 3.658643000 & -1.026180000 \\
1 & -0.696095000 & 1.178962000 & 2.511795000 & 1 & -0.690238000 & 1.142180000 & 2.516876000 \\
1 & 0.611063000 & 0.747508000 & 0.914742000 & 1 & 0.561609000 & 0.759452000 & 0.880120000 \\
1 & -0.412024000 & 1.382854000 & -2.238437000 & 1 & -0.550104000 & 1.422110000 & -2.237008000 \\
1 & -3.003532000 & 0.037636000 & -1.421534000 & 1 & -3.073289000 & -0.038366000 & -1.351637000 \\
1 & -3.139310000 & -0.318071000 & 1.237363000 & 1 & -3.111637000 & -0.433517000 & 1.304890000 \\
1 & -1.049290000 & -1.578719000 & -2.382273000 & 1 & -1.086726000 & -1.562321000 & -2.394915000 \\
1 & -1.306368000 & -2.179708000 & 1.931103000 & 1 & -1.181539000 & -2.224124000 & 1.916387000 \\
1 & -0.002862000 & -2.918268000 & -0.304640000 & 1 & 0.081005000 & -2.880306000 & -0.368457000 \\
6 & 1.421698000 & -0.057308000 & 0.219110000 & 6 & 2.295918000 & 0.580713000 & -0.733869000 \\
6 & 2.202601000 & -0.937935000 & 0.941524000 & 6 & 1.423799000 & 0.002035000 & 0.151421000 \\
6 & 3.513094000 & -0.915585000 & 0.383024000 & 6 & 2.231001000 & -0.946686000 & 0.893133000 \\
6 & 3.461377000 & -0.006057000 & -0.634815000 & 6 & 3.488515000 & -0.873982000 & 0.387226000 \\
8 & 2.223552000 & 0.528011000 & -0.744923000 & 8 & 3.545869000 & 0.058948000 & -0.613064000 \\
1 & 1.865212000 & -1.525228000 & 1.784158000 & 1 & 2.174287000 & 1.343313000 & -1.485767000 \\
1 & 4.376633000 & -1.485465000 & 0.695233000 & 1 & 1.900182000 & -1.588540000 & 1.697273000 \\
1 & 4.197514000 & 0.359126000 & -1.335785000 & 1 & 4.415298000 & -1.381787000 & 0.605868000 \\
\hline
\end{tabular}

